\title{
Differentiable invariant manifolds for partially hyperbolic tori and a lambda lemma
}

\author{
E Fontich* \\ Departament de Matemàtica Aplicada i Anàlisi \\ Universitat de Barcelona \\ Gran Via, 585, 08007 Barcelona, Spain \\ $P$ Martín $^{\dagger}$ \\ Departament de Matemàtica Aplicada i Telemàtica \\ Universitat Politècnica de Catalunya \\ Ed-C3, Jordi Girona, 1-3, 08034 Barcelona Spain
}

\begin{abstract}
We consider differentiable maps having an invariant torus with normal behavior having a central part. We prove the existence and regularity of pseudostable manifolds and regularity with respect to parameters. Then we prove a lambda lemma in this setting for $C^{2}$ maps.
\end{abstract}

AMS Subject Classification (1991): 58F30, 34C35, 34C37.

\section{Introduction}

In the context of Hamiltonian dynamics, small perturbations of integrable Hamiltonian systems preserve, slightly deformed, a large set of invariant tori of maximal dimension (equal to the number of degrees of freedom). The tori with more that one ergodic component are generically destroyed. However, some of their ergodic components may survive, giving rise to the existence of lower dimensional tori.

The persistence of lower dimensional tori, when they come from non-degenerate and partially hyperbolic tori of the non-perturbed system, can be found, for instance in [11], [14], [19] in the analytic setting. Tori coming from the breakdown of maximal invariant tori are studied in [17], see also [8]. Moreover, these lower dimensional tori have invariant manifolds related to the their hyperbolic directions.

In general the existence of these tori can be stated independently of the existence of their invariant manifolds (this fact also happens when dealing with normally hyperbolic manifolds or, in general, when studying the persistence of invariant objects in dynamics). From the persistence under perturbation of the object, it follows that it can be considered fixed by the perturbation, and its existence taken as a hypothesis. Then, the existence and other

\footnotetext{
*fontich@mat.ub.es

${ }^{\dagger}$ martin@mat.upc.es
} 
properties of its related invariant manifolds can be studied. We follow this approach, since the phenomena involved in the persistence of the tori differ from the ones involved in the existence of its invariant manifolds. In particular, as far as the symplectic structure plays no significant role in the existence of the invariant manifolds, we shall consider partially hyperbolic tori of systems, which include a general integrable system, and perturbations which vanish on the tori. In many applications, this is not restrictive in the sense that the system can be written in the form we assume.

One of the goals of this paper is to study the existence and regularity of invariant manifolds of tori for families of maps, when the normal behavior has both a hyperbolic and a central part. These tori appear in perturbations of integrable symplectic maps, as well as Poincaré maps and $\tau$-maps of Hamiltonian systems. Although in the symplectic context these invariant manifolds may have interesting additional properties we work with non necessarily symplectic maps. Also we study the dependence on parameters.

We consider maps $F_{\nu}(w)$ defined on $\mathcal{M}_{\delta} \subset \mathcal{M}$ where $\mathcal{M}=\mathbb{R}^{m} \times \mathbb{R}^{p} \times \mathbb{T}^{n} \times \mathbb{R}^{q}, \mathcal{M}_{\delta}=$ $\mathcal{B}_{\delta}^{m} \times \mathcal{B}_{\delta}^{p} \times \mathbb{T}^{n} \times \mathcal{B}_{\delta}^{q}$, and $\mathbb{T}=\mathbb{R} / \mathbb{Z}, \nu \in \mathcal{B}_{\mu}^{s} \subset \mathbb{R}^{s}$. We consider perturbations of

$$
F_{\nu}(w)=\left(A_{-}(\theta) x, A_{+}(\theta) y, \theta+\omega(x, y, r), B(\theta) r\right)
$$

and we assume that $\left\|A_{-}(\theta)\right\| \leq \lambda^{-1}<1, A_{+}(\theta)$ and $B(\theta)$ are invertible and $\left\|A_{+}(\theta)^{-1}\right\| \leq \lambda^{-1}$ and $\left\|B(\theta)^{-1}\right\| \leq \beta$. If $f=0$ the set $\{y=r=0\}$ is an stable invariant manifold, $y$ is an unstable direction and $r$ may be a central direction, although it can contain unstable directions as well as stable ones. When $f \neq 0$ we look for the nonlinear version of $\{y=0, r=$ $0\}$. That is we look for an invariant manifold parametrized by $(x, \theta)$. In fact the variables $y, r$ will play a similar role and will be considered as a unique variable $v$. Also $A_{+}, B$ will considered together.

This formulation permits to take different decompositions of $w$ as $(x, y, \theta, r)$. For instance if $A_{-}=\operatorname{diag}\left(A_{1-}, A_{2-}\right)$ with $\left\|A_{1-}\right\| \leq \lambda_{1}^{-1}<\lambda^{-1}$ and $x=\left(x_{1}, x_{2}\right)$ according to this decomposition, we can look for invariant manifolds parametrized by $\left(x_{1}, \theta\right)$ considering $x_{2}$ as part of $(y, r)$ and $A_{2-}$ as part of $\operatorname{diag}\left(A_{+}, B\right)$.

We permit perturbations $f=\left(f_{1}, f_{2}, f_{3}, f_{4}\right)$ such that $f_{j}, j=1,2,4$, have terms of order strictly bigger than 1 in $(x, y, r)$ or terms of order 1 in $(x, y, r)$, going to zero as $\nu \rightarrow 0$. See hypothesis H3. We permit some non-trivial dynamics on the torus. The main condition we ask is that $f_{3}$ verifies that if $K_{\theta}=\sup \left\|\partial_{\theta} f_{3}(w)\right\|$ then $\lambda^{-1} \beta\left(1+K_{\theta}\right)^{k}<1$ where $k$ is the degree of differentiability we want to obtain for the invariant manifold. In fact we can let $A_{ \pm}$and $B$ depend on $\nu$ in some uniform way. First we consider the case of Lipschitz perturbations and we get, under some natural conditions, that the tori have a local stable invariant manifold as a graph of a Lipschitz function. Then we consider the $C^{k}$ case under hypotheses $\mathrm{HC} 1$ to $\mathrm{HC} 4$, and finally the case $C^{\infty}$. In this later case the hypotheses HI1 to HI4 imply that the dynamics on the torus is restricted to be a rotation. However we do not impose any condition on the frequency vector of the rotation. The analytic case is also considered in [10].

We produce the stable manifold. We note that we get the stable manifold (as it is the case for fixed points) for not necessarily invertible maps. The results for the unstable one readily follow from the stable under suitable invertibility conditions. See the beginning of Section 7 .

Uniqueness is discussed in Remark 2.4. 
In Section 7 we produce a version of the lambda lemma in the analogous setting we have considered to study the invariant manifolds for maps of class $C^{2}$. We obtain the $C^{1}$ convergence of transversal manifolds to the stable manifold to the unstable one.

The main difference with the original lambda lemma [16] stays in the control of the variables associated to the central directions as well as the control of the angles. In [18] there is a lambda lemma for normally hyperbolic tori. For analytic maps, there is a very similar result in [15] where $(n-1)$-tori are considered and a recent work [4] considers the case of $(n-k)$-partially hyperbolic invariant tori.

These results are relevant in the study of diffusion problems, where some non analytical objects have to be considered as it may be the case when one considers variational methods. In particular in Arnold's diffusion $[1,3,6,7,9]$. Using invariant manifolds of invariant objects and a lambda lemma one can find, in some systems, orbits of unbounded energy [2, 5].

\section{The Lipschitz case}

Let $\mathcal{M}$ and $\mathcal{M}_{\delta}$ be the sets $\mathcal{M}=\mathbb{R}^{m} \times \mathbb{R}^{p} \times \mathbb{T}^{n} \times \mathbb{R}^{q}, \mathcal{M}_{\delta}=\mathcal{B}_{\delta}^{m} \times \mathcal{B}_{\delta}^{p} \times \mathbb{T}^{n} \times \mathcal{B}_{\delta}^{q}$, where $\mathbb{T}=\mathbb{R} / \mathbb{Z}$, and $\mathcal{B}_{\delta}^{j}$ is the ball of radius $\delta$ in $\mathbb{R}^{j}$. Let $F_{\nu}: \mathcal{M}_{\delta} \rightarrow \mathcal{M}, \nu \in \mathcal{B}_{\mu}^{s} \subset \mathbb{R}^{s}$, be a family of maps of the form

$$
F_{\nu}(w)=\left(A_{-}(\theta) x, A_{+}(\theta) y, \theta+\omega(x, y, r), B(\theta) r\right)+f(w, \nu)
$$

where $w=(x, y, \theta, r), A_{-}(\theta) \in L\left(\mathbb{R}^{m}, \mathbb{R}^{m}\right), A_{+}(\theta) \in L\left(\mathbb{R}^{p}, \mathbb{R}^{p}\right), B(\theta) \in L\left(\mathbb{R}^{q}, \mathbb{R}^{q}\right)$ and $f=\left(f_{1}, f_{2}, f_{3}, f_{4}\right)$. We assume that there exist positive constants $\lambda, \beta$ and $K_{\theta}$ such that

$$
\lambda>1, \quad \beta \geq 1, \quad \lambda^{-1} \beta\left(1+K_{\theta}\right)<1
$$

and a non-negative continuous function $L(\delta, \mu)$ with $L(0,0)=0$ such that the following hypotheses hold

H1 The functions $A_{ \pm}, B, \omega$ and $f$ are Lipschitz with respect to $w$.

H2 The functions $A_{ \pm}$and $B$ verify

$$
\left\|A_{-}(\theta)\right\| \leq \lambda^{-1}, \quad\left\|A_{+}(\theta)^{-1}\right\| \leq \lambda^{-1}, \quad\left\|B^{-1}(\theta)\right\| \leq \beta, \quad \forall \theta \in \mathbb{T}^{n} .
$$

H3 For $j=1,2$, 4 , the functions $f_{j}$ are $L(\delta, \mu)$-Lipschitz with respect to $w$, satisfy $f_{j}(0,0, \theta, 0, \nu)=$ 0 , and

$$
\left\|f_{j}(x, y, \theta, r, \nu)-f_{j}\left(x, y, \theta^{\prime}, r, \nu\right)\right\| \leq K_{m}\|(x, y, r)\|\left\|\theta-\theta^{\prime}\right\| .
$$

H4 The function $f_{3}$ is $K_{\theta}$-Lipschitz with respect to $\theta$.

Apart from the different constants just introduced we call

$$
K_{\omega}=\operatorname{Lip} \omega, \quad K_{l}=\max \left(\operatorname{Lip} A_{-}, \operatorname{Lip} A_{+}, \operatorname{Lip} B\right), \quad K_{f}=\operatorname{Lip} f_{3} .
$$

The condition that $f_{j}$ are $L(\delta, \mu)$-Lipschitz must be considered as that $f_{j}$ restricted to $\mathcal{M}_{\delta}$ with $\nu \in \mathcal{B}_{\mu}^{s}$ have Lipschitz constant $L(\delta, \mu)$. Therefore, if we consider $\delta$ and $\mu$ small enough, the Lipschitz constant will become smaller.

We are interested in the invariant manifolds of $\mathbb{T}_{0}=\{x=0, y=0, r=0\}$ which, under the preceding hypotheses, is a torus invariant by $F_{\nu}$. 
Theorem 2.1 Let $F_{\nu}: \mathcal{M}_{\delta} \rightarrow \mathcal{M}$ satisfy hypotheses $H_{1}$ to $H_{4}$. Then there exist $\delta_{0}$ and $\mu_{0}$ such that, if $0<\delta<\delta_{0}$ and $\mu \in \mathcal{B}_{\mu_{0}}^{s}$, the invariant torus $\mathbb{T}_{0}$ of the map $F_{\nu}$ defined by (2.1) has an invariant manifold which can be represented as the graph of a Lipschitz function $\gamma_{\nu}^{s}(x, \theta)$, which verifies $\gamma_{\nu}^{s}(0, \theta)=0$. Its Lipschitz constant is of order $\delta+L(\delta, \mu)$. The iterates of the points $\left(x, \theta, \gamma_{\nu}^{s}(x, \theta)\right)$ tend to the torus, exponentially fast. Moreover if we ask

H5 For $j=1,2,4,\left\|f_{j}(x, y, \theta, r, \nu)-f_{j}\left(x, y, \theta, r, \nu^{\prime}\right)\right\| \leq K_{\mu}\|(x, y, r)\|\left\|\nu-\nu^{\prime}\right\|$ and $f_{3}$ is $K_{\theta}$-Lipschitz with respect to $\nu$.

then $\gamma_{\nu}^{s}$ depends in a Lipschitz way on $\nu$.

Remark 2.2 We do not ask $F$ to be invertible. For $F$ invertible we can get the existence of an unstable invariant manifold by just considering $F^{-1}$. A set of sufficient conditions for $F$ to be invertible and its inverse to have the same form is that $K_{\theta}<1$ and the bounds $\|B(\theta)\| \leq \beta,\left\|A_{-}(\theta)^{-1}\right\| \geq \lambda,\left\|A_{+}(\theta)\right\| \geq \lambda$ for $\theta \in \mathbb{T}^{n}$.

In fact, a similar result can be obtained if we do not assume any particular form for the third component of $F$ but only a Lipschitz condition on it and that $F$ is invertible, the inverse having the same form.

Remark 2.3 We may let $A_{ \pm}$and $B$ depend on $\nu$ in such a way that hypotheses $H 1$ and $H 2$ hold uniformly in $\nu$ and that they are Lipschitz in $\nu$. However terms linear in $(x, y, r)$ going to zero as $\nu$ goes to zero can be included in $f$.

Remark 2.4 The set of points which tend to the torus may be a complicated set. For instance, it may have dimension $m+n+q$ as the example given by $B(\theta)=I, f_{1}=0, f_{2}=0$, $f_{3}=0, f_{4}=-r^{3}$ shows. However, the invariant manifolds given by Theorem 2.1 are unique in the following sense: consider the space

$$
\Sigma_{c, M}=\left\{\gamma \in \Sigma ; \operatorname{lip} \gamma \leq c,\left\|\gamma(x, \theta)-\gamma\left(x, \theta^{\prime}\right)\right\| \leq M\|x\|\left\|\theta-\theta^{\prime}\right\|\right\},
$$

where $\Sigma$ is the Banach space defined below in (2.6). From the proof of Theorem 2.1 we have that, taking $c \leq 1$ and $M$ large enough, there is a function, $\gamma^{s} \in \Sigma_{c, M}$ whose graph represents the stable manifold of $F$. But, in fact, for all $c, M$ such that

$$
c<\frac{1-\beta \lambda^{-1}}{2 \beta L}-2, \quad M<\frac{1-\beta \lambda^{-1}}{2 \beta\left(\lambda^{-1}+L\right)\left(K_{\omega}+K_{f}\right)} \frac{1}{\delta},
$$

$\gamma^{s}$ is the only function satisfying the invariance condition in $\Sigma_{c, M}$. Notice that $c=O\left(L^{-1}\right)$ and $M=O\left(\delta^{-1}\right)$ (see below, at the end of the section, the proof of the remark to obtain other possible choices for the bounds of $c$ and $M$.)

Notation. To simplify the notation we shall not explicitly write the dependence of the involved functions on $\nu$. We shall write $L$ instead of $L(\delta, \mu)$ but we must keep in mind its dependence on $\delta$ and $\mu$. We shall group the variables in $z=(x, \theta)$ and $v=(y, r)$. We assume that on $\mathbb{R}^{m}, \mathbb{R}^{p}, \mathbb{R}^{n}$ and $\mathbb{R}^{q}$ there are defined some norms. In any product space we take the maximum norm, for instance $\|(x, y, r)\|=\max (\|x\|,\|y\|,\|r\|)$.

In general, for functions $\psi$ defined on $\mathcal{B}_{\delta}^{m} \times \mathbb{T}^{n}$ and taking values on some space we write

$$
\|\psi\|_{0}=\sup _{(x, \theta) \in \mathcal{B}_{\delta}^{m} \times \mathbb{T}^{n}}\|\psi(x, \theta)\|, \quad\|\psi\|_{1}=\sup _{(x, \theta) \in \mathcal{B}_{\delta}^{m} \times \mathbb{T}^{n}, x \neq 0}\|x\|^{-1}\|\psi(x, \theta)\| .
$$


We shall denote

$$
g=-\left(f_{2}, f_{4}\right)^{t}
$$

Note that $g$ also verifies hypothesis H3. Also, we shall denote $A_{-}$by $A$. We define

$$
C(\theta)=\left(\begin{array}{cc}
A_{+}(\theta) & 0 \\
0 & B(\theta)
\end{array}\right)
$$

and, in accordance with the choice of the norms, $\|C(\theta)\|=\max \left\{\left\|A_{+}(\theta)\right\|,\|B(\theta)\|\right\}$. Consequently, $\left\|C(\theta)^{-1}\right\| \leq \beta$ and $C(\theta)^{-1}$ is $K_{l} \beta^{2}$-Lipschitz. Indeed,

$$
\left\|C(\theta)^{-1}-C\left(\theta^{\prime}\right)^{-1}\right\| \leq\left\|C(\theta)^{-1}\right\|\left\|C\left(\theta^{\prime}\right)^{-1}\right\|\left\|C(\theta)-C\left(\theta^{\prime}\right)\right\| \leq K_{l} \beta^{2}\left\|\theta-\theta^{\prime}\right\| .
$$

We shall look for the stable manifold of $\mathbb{T}_{0}$ as a graph of a function of $(x, \theta)$ which is invariant by $F$. The invariance condition for graph $\gamma$ is

$$
\pi_{y, r} F(z, \gamma(z))=\gamma\left(\pi_{x, \theta} F(z, \gamma(z))\right)
$$

where $\pi_{s}$ denotes a projector to the subspace spanned by the variables $s$, or equivalently

$$
\gamma(z)=C(\theta)^{-1}\left(g(z, \gamma(z))+\gamma\left(A(\theta) x+f_{1}(z, \gamma(z)), \theta+\omega(x, \gamma(z))+f_{3}(z, \gamma(z))\right)\right)
$$

We introduce $h(\gamma)=\left(h_{1}(\gamma), h_{2}(\gamma)\right)^{t}$ where

$$
\begin{aligned}
& h_{1}(z)=h_{1}(\gamma)(z)=A(\theta) x+f_{1}(z, \gamma(z)) \\
& h_{2}(z)=h_{2}(\gamma)(z)=\theta+\omega(x, \gamma(z))+f_{3}(z, \gamma(z)) .
\end{aligned}
$$

When working with a fixed $\gamma$, without danger of confusion, we shall simply write $h_{j}$ or $h_{j}(z)$ instead of $h_{j}(\gamma)(z)$.

Therefore we define $G$ by

$$
G(\gamma)(z)=C(\theta)^{-1}(g(z, \gamma(z))+\gamma(h(\gamma)(z)))
$$

We introduce

$$
\Sigma=\left\{\gamma: \mathcal{B}_{\delta}^{m} \times \mathbb{T}^{n} \rightarrow \mathbb{R}^{p} \times \mathbb{R}^{q} ; \gamma \text { is continuous },\|\gamma\|_{1}<\infty\right\},
$$

which is a Banach space with the norm $\|\gamma\|_{1}$, and, given $M>0, c>0$, the closed subset

$$
\Sigma_{c, M}=\left\{\gamma \in \Sigma ; \text { Lip } \gamma \leq c,\left\|\gamma(x, \theta)-\gamma\left(x, \theta^{\prime}\right)\right\| \leq M\|x\|\left\|\theta-\theta^{\prime}\right\|\right\} .
$$

Notice that if $\gamma \in \Sigma_{c, M}$ we have that

$$
\gamma(0, \theta)=0, \quad\|\gamma(z)\|=\|\gamma(x, \theta)-\gamma(0, \theta)\| \leq c\|x\| .
$$

Proof of Theorem 2.1 To prove the existence of the stable manifold we have to solve the fixed point equation $\gamma=G(\gamma)$. We shall prove that actually $G$ is a well defined map from $\Sigma_{c, M}$ to itself and that it is a contraction. Such a claim will be a consequence of the next lemmas. First we state some useful bounds. 
From now on, $c, M, \delta$ and $\mu$ will be positive constants that we will fix later on, and $0<c \leq 1$. Using H3, H4 we easily get that for $\gamma \in \Sigma_{c, M}$

$$
\begin{aligned}
\left\|f_{j}(z, \gamma(z))\right\| & \leq L\|x\|, \quad j=1,2,4 \\
\left\|f_{j}(z, \gamma(z))-f_{j}\left(z^{\prime}, \gamma\left(z^{\prime}\right)\right)\right\| & \leq L\left\|z-z^{\prime}\right\|, \quad j=1,2,4 \\
\left\|f_{j}(x, \theta, \gamma(x, \theta))-f_{j}\left(x, \theta^{\prime}, \gamma\left(x, \theta^{\prime}\right)\right)\right\| & \leq\left(K_{m}+L M\right)\|x\|\left\|\theta-\theta^{\prime}\right\|, \quad j=1,2,4,(2.1 \\
\left\|f_{3}(z, \gamma(z))-f_{3}\left(z^{\prime}, \gamma\left(z^{\prime}\right)\right)\right\| & \leq K_{f}\left\|z-z^{\prime}\right\| .
\end{aligned}
$$

Note that $g$ also satisfies (2.9), (2.10) and (2.11).

Let $\gamma \in \Sigma_{c, M}$ and $z, z^{\prime},\left(x, \theta^{\prime}\right) \in \mathcal{B}_{\delta}^{m} \times \mathbb{T}^{n}$. From H2, H3 and the previous bounds we easily get

$$
\begin{aligned}
\left\|h_{1}(z)\right\| & \leq\left(\lambda^{-1}+L\right)\|x\|, \\
\left\|h_{1}(z)-h_{1}\left(z^{\prime}\right)\right\| & \leq\left(\lambda^{-1}+L+K_{l} \delta\right)\left\|z-z^{\prime}\right\| \\
\left\|h_{1}(x, \theta)-h_{1}\left(x, \theta^{\prime}\right)\right\| & \leq\left(K_{l}+K_{m}+L M\right)\|x\|\left\|\theta-\theta^{\prime}\right\| \\
\left\|h_{2}(z)-h_{2}\left(z^{\prime}\right)\right\| & \leq\left(1+K_{\omega}+K_{f}\right)\left\|z-z^{\prime}\right\| \\
\left\|h_{2}(x, \theta)-h_{2}\left(x, \theta^{\prime}\right)\right\| & \leq\left(1+K_{\theta}+\left(K_{\omega}+K_{f}\right) M\|x\|\right)\left\|\theta-\theta^{\prime}\right\| .
\end{aligned}
$$

From (2.13) we have that if $\delta$ and $\mu$ are small enough so that $\lambda^{-1}+L<1$, then $h(\gamma)$ maps $\mathcal{B}_{\delta}^{m} \times \mathbb{T}^{n}$ to itself. In particular, $G(\gamma)$ is well defined on $\mathcal{B}_{\delta}^{m} \times \mathbb{T}^{n}$.

Lemma 2.5 For any $c \in(0,1]$ and $M$ such that

$$
M>\frac{\beta K_{m}+c\left(K_{l}+K_{m}+K_{l} \beta \lambda^{-1}\right)}{1-\beta \lambda^{-1}\left(1+K_{\theta}\right)}
$$

there exists $\delta_{0}, \mu_{0}$ such that, for all $\delta \in\left(0, \delta_{0}\right], \mu \in \mathcal{B}_{\mu_{0}}^{s}, G\left(\Sigma_{c, M}\right) \subset \Sigma_{c, M}$.

Proof of Lemma 2.5. Clearly $G(\gamma)$ is continuous. By (2.9), (2.8) and (2.13) we have

$$
\|g(z, \gamma(z))+\gamma(h(z))\| \leq L\|x\|+c\left\|h_{1}(z)\right\| \leq\left(L+c\left(\lambda^{-1}+L\right)\right)\|x\|
$$

and then $\|x\|^{-1}\|G(\gamma)(z)\| \leq \beta\left(L+c\left(\lambda^{-1}+L\right)\right)$.

Next we bound

$$
\begin{aligned}
& \left\|\gamma(h(z))-\gamma\left(h\left(z^{\prime}\right)\right)\right\| \\
& \quad \leq\left\|\gamma\left(h_{1}(z), h_{2}(z)\right)-\gamma\left(h_{1}(z), h_{2}\left(z^{\prime}\right)\right)\right\|+\left\|\gamma\left(h_{1}(z), h_{2}\left(z^{\prime}\right)\right)-\gamma\left(h_{1}\left(z^{\prime}\right), h_{2}\left(z^{\prime}\right)\right)\right\| \\
& \quad \leq M\left\|h_{1}(z)\right\|\left\|h_{2}(z)-h_{2}\left(z^{\prime}\right)\right\|+c\left\|h_{1}(z)-h_{1}\left(z^{\prime}\right)\right\| .
\end{aligned}
$$

Then using (2.20) and (2.19) we obtain that

$$
\begin{aligned}
& \left\|G(\gamma)(z)-G(\gamma)\left(z^{\prime}\right)\right\| \\
& \leq \quad\left\|C(\theta)^{-1}\right\|\left\|g(z, \gamma(z))-g\left(z^{\prime}, \gamma\left(z^{\prime}\right)\right)+\gamma(h(z))-\gamma\left(h\left(z^{\prime}\right)\right)\right\| \\
& \quad+\left\|C(\theta)^{-1}-C\left(\theta^{\prime}\right)^{-1}\right\|\left\|g\left(z^{\prime}, \gamma\left(z^{\prime}\right)\right)+\gamma\left(h\left(z^{\prime}\right)\right)\right\| \\
& \leq \quad \beta\left(c \lambda^{-1}+\delta\left(M\left(\lambda^{-1}+L\right)\left(1+K_{\omega}+K_{f}\right)+c K_{l}\right)+L(1+c)\right)\left\|z-z^{\prime}\right\| \\
& \quad+K_{l} \beta^{2}\left(L+c\left(\lambda^{-1}+L\right)\right) \delta\left\|\theta-\theta^{\prime}\right\| .
\end{aligned}
$$


This implies that $G(\gamma)$ is $c$-Lipschitz because the coefficient of $\left\|z-z^{\prime}\right\|$ tend to $\beta \lambda^{-1} c<c$ when $\delta, \mu \rightarrow 0$.

It remains to be proved that $\left\|G(\gamma)(x, \theta)-G(\gamma)\left(x, \theta^{\prime}\right)\right\| \leq M\|x\|\left\|\theta-\theta^{\prime}\right\|$. We write $\tilde{z}=$ $\left(x, \theta^{\prime}\right)$. From $(2.20),(2.13),(2.15)$ and $(2.17)$

$$
\begin{aligned}
& \|\gamma(h(z))-\gamma(h(\tilde{z}))\| \leq M\left\|h_{1}(z)\right\|\left\|h_{2}(z)-h_{2}(\tilde{z})\right\|+c\left\|h_{1}(z)-h_{1}(\tilde{z})\right\| \\
& \quad \leq\left(M\left(\lambda^{-1}+L\right)\left(1+K_{\theta}+\left(K_{\omega}+K_{f}\right) M \delta\right)+c\left(K_{l}+K_{m}+L M\right)\right)\|x\|\left\|\theta-\theta^{\prime}\right\| .(2 .)
\end{aligned}
$$

Using (2.11), (2.22) and (2.19)

$$
\begin{aligned}
& \|G(\gamma)(z)-G(\gamma)(\tilde{z})\| \\
& \leq \quad \beta\|g(z, \gamma(z))-g(\tilde{z}, \gamma(\tilde{z}))\|+\beta\|\gamma(h(z))-\gamma(h(\tilde{z}))\| \\
& \quad+K_{l} \beta^{2}\|g(\tilde{z}, \gamma(\tilde{z}))+\gamma(h(\tilde{z}))\|\left\|\theta-\theta^{\prime}\right\| \\
& \leq \quad\left(\beta\left(K_{m}+L M\right)+\beta\left(M\left(\lambda^{-1}+L\right)\left(1+K_{\theta}+\left(K_{\omega}+K_{f}\right) M \delta\right)+c\left(K_{l}+K_{m}+L M\right)\right)\right. \\
& \left.\quad+K_{l} \beta^{2}\left(L+c\left(\lambda^{-1}+L\right)\right)\right)\|x\|\left\|\theta-\theta^{\prime}\right\| .
\end{aligned}
$$

The result follows because the coefficient of $\|x\|\left\|\theta-\theta^{\prime}\right\|$ tends to $\beta\left(K_{m}+M \lambda^{-1}\left(1+K_{\theta}\right)+\right.$ $\left.c\left(K_{l}+K_{m}\right)+K_{l} \beta c \lambda^{-1}\right)$ as $\delta, \mu \rightarrow 0$.

Lemma 2.6 Let $c \in(0,1]$ and $M$ verifying (2.18). Then, there exist $\delta_{0}$ and $\mu_{0}$ such that for all $\delta \in\left(0, \delta_{0}\right], \mu \in \mathcal{B}_{\mu_{0}}^{s}$, the map $G: \Sigma_{c, M} \rightarrow \Sigma_{c, M}$ is a contraction.

Proof of Lemma 2.6. Notice that $\left\|h_{1}(\gamma)(z)-h_{1}(\tilde{\gamma})(z)\right\|=\left\|f_{1}(z, \gamma(z))-f_{1}(z, \tilde{\gamma}(z))\right\| \leq$ $L\|\gamma(z)-\tilde{\gamma}(z)\|$ and $\left\|h_{2}(\gamma)(z)-h_{2}(\tilde{\gamma})(z)\right\| \leq\|\omega(x, \gamma(z))-\omega(x, \tilde{\gamma}(z))\|+\left\|f_{3}(z, \gamma(z))-f_{3}(z, \tilde{\gamma}(z))\right\|$ $\leq\left(K_{\omega}+K_{f}\right)\|\gamma(z)-\tilde{\gamma}(z)\|$. From

$$
\begin{aligned}
& \|\gamma(h(\gamma)(z))-\gamma(h(\tilde{\gamma})(z))\| \\
& \quad \leq\left\|\gamma(h(\gamma)(z))-\gamma\left(h_{1}(\gamma)(z), h_{2}(\tilde{\gamma})(z)\right)\right\|+\left\|\gamma\left(h_{1}(\gamma)(z), h_{2}(\tilde{\gamma})(z)\right)-\gamma(h(\tilde{\gamma})(z))\right\| \\
& \quad \leq M\left(\lambda^{-1}+L\right)\left(K_{\omega}+K_{f}\right)\|x\|^{2}\|\gamma-\tilde{\gamma}\|_{1}+c L\|x\|\|\gamma-\tilde{\gamma}\|_{1}
\end{aligned}
$$

we have

$$
\begin{aligned}
& \|\gamma(h(\gamma)(z))-\tilde{\gamma}(h(\tilde{\gamma})(z))\| \leq\|\gamma(h(\gamma)(z))-\gamma(h(\tilde{\gamma})(z))\|+\|\gamma(h(\tilde{\gamma})(z))-\tilde{\gamma}(h(\tilde{\gamma})(z))\| \\
& \quad \leq\left[M\left(\lambda^{-1}+L\right)\left(K_{\omega}+K_{f}\right)\|x\|+c L+\lambda^{-1}+L\right]\|x\|\|\gamma-\tilde{\gamma}\|_{1} .
\end{aligned}
$$

Then

$$
\begin{aligned}
& \|G(\gamma)(z)-G(\tilde{\gamma})(z)\| \leq \beta(\|g(z, \gamma(z))-g(z, \tilde{\gamma}(z))\|+\|\gamma(h(\gamma)(z))-\tilde{\gamma}(h(\tilde{\gamma})(z))\|) \\
& \quad \leq \beta\left(L\|\gamma(z)-\tilde{\gamma}(z)\|+\left(M\left(\lambda^{-1}+L\right)\left(K_{\omega}+K_{f}\right) \delta+\lambda^{-1}+L(1+c)\right)\|x\|\|\gamma-\tilde{\gamma}\|_{1}\right) \\
& \quad \leq\left[\beta \lambda^{-1}+O(L)+O(\delta)\right]\|x\|\|\gamma-\tilde{\gamma}\|_{1} .
\end{aligned}
$$

End of the proof of Theorem 2.1. By lemmas 2.5 and 2.6, if $c \in(0,1]$ and $M$ verifies (2.18), $G: \Sigma_{c, M} \longrightarrow \Sigma_{c, M}$ is a well defined contraction. Once we have fixed $\delta$ and $\mu$ small 
enough so that the previous lemmas hold we can ask which is the minimum value we can take for $c$. It appears that this choice is limited by the bound in (2.21) and from this we get that we can take $c=O(\delta+L)$. Moreover it is clear that the iterates of $\left(z, \gamma^{s}(z)\right)$ tend to the torus because $\left\|F_{1}\left(z, \gamma^{s}(z)\right)\right\| \leq\left(\lambda^{-1}+L\right)\|x\|$.

To study the dependence on $\nu$ we introduce the map $\tilde{F}$ defined by $\tilde{F}(w, \nu)=(F(w, \nu), \nu)$. Since $\nu \in \mathcal{B}_{\mu}^{s} \subset \mathbb{R}^{s}$, where $\mu$ is small enough in order to apply the former arguments, we can consider $\nu \in \mathbb{T}^{s}$, extending the function $F$ outside $\mathcal{B}_{\mu}^{s}$ in a Lipschitz periodic manner keeping the bounds in $\mathrm{H} 1$ to $\mathrm{H} 4$. That is, we can consider the parameters as angles. Hence, we obtain that $\tilde{F}$ has an invariant manifold parameterized by a Lipschitz function $\gamma(x, \theta, \nu)$, which for a fixed value of $\nu$, is the invariant manifold of $F_{\nu}$.

Proof of Remark 2.4. Let $\gamma$ and $\tilde{\gamma}$ be two functions in $\Sigma_{c, M}$ verifying the invariance condition, that is, using the previous notations, $\gamma(z)=C(\theta)^{-1}(g(z, \gamma(z))+\gamma(h(\gamma)(z)))$ and $\tilde{\gamma}(z)=C(\theta)^{-1}(g(z, \tilde{\gamma}(z))+\tilde{\gamma}(h(\tilde{\gamma})(z)))$. To simplify the notation we introduce $z^{*}=\left(x^{*}, \theta^{*}\right)=$ $h(\gamma)(z)$ and $\tilde{z}^{*}=\left(\tilde{x}^{*}, \tilde{\theta}^{*}\right)=h(\tilde{\gamma})(z)$. Subtracting we can apply the bound in $(2.23)$

$$
\begin{aligned}
\|\gamma(z)-\tilde{\gamma}(z)\| & \leq\left\|C^{-1}\right\|\left(\left\|\gamma\left(z^{*}\right)-\tilde{\gamma}\left(\tilde{z}^{*}\right)\right\|+\|g(z, \gamma(z))-g(z, \tilde{\gamma}(z))\|\right) \\
& \leq \beta\left(\lambda^{-1}+M\left(\lambda^{-1}+L\right)\left(K_{\omega}+K_{f}\right) \delta+L(2+c)\right)\|x\|\|\gamma-\tilde{\gamma}\|_{1} .
\end{aligned}
$$

Therefore, dividing by $\|x\|$ and taking the supremum with respect to $x$ we finally obtain

$$
\|\gamma-\tilde{\gamma}\|_{1} \leq \beta\left(\lambda^{-1}+M\left(\lambda^{-1}+L\right)\left(K_{\omega}+K_{f}\right) \delta+L(2+c)\right)\|\gamma-\tilde{\gamma}\|_{1} .
$$

From the conditions on $c$ and $M$ stated in the remark we have that the coefficient of $\|\gamma-\tilde{\gamma}\|_{1}$ in the right hand side is strictly less than 1 , which implies $\gamma=\tilde{\gamma}$.

\section{The $C^{k}$ Case}

In this section we consider the $C^{k}$ case. We consider again the family of maps $F_{\nu}: \mathcal{M}_{\delta} \longrightarrow$ $\mathcal{M}$ defined by (2.1) assuming differentiability conditions. We consider the following set of hypotheses which are, essentially, the differentiable version of the hypotheses assumed in the Lipschitz case. Let $\lambda, \beta$ and $K_{\theta}$ such that

$$
\lambda>1, \quad \beta \geq 1, \quad \lambda^{-1} \beta\left(1+K_{\theta}\right)^{k}<1
$$

and let $L(\delta, \mu)$ be as in the previous section, verifying

HC1 The functions $A_{ \pm}, B, \omega$ and $f$ are $C^{k}$ both with respect to $w$ and $\nu$.

HC2 $A_{-}, A_{+}$and $B$ verify

$$
\left\|A_{-}(\theta)\right\| \leq \lambda^{-1}, \quad\left\|A_{+}(\theta)^{-1}\right\| \leq \lambda^{-1}, \quad\left\|B^{-1}(\theta)\right\| \leq \beta, \quad \forall \theta \in \mathbb{T}^{n} .
$$

HC3 For $j=1,2,4$ the functions $f_{j}$ have the form

$$
f_{j}(w, \nu)=\varphi_{j 1}(w, \nu) x+\varphi_{j 2}(w, \nu) v, \quad v=(y, r),
$$

with $\varphi_{j i}$ of class $C^{k}$ with respect to $\theta$, and

$$
\left\|\partial_{\theta} f_{j}(w, \nu)\right\| \leq K_{m}\|(x, y, r)\|, \quad\left\|D f_{j}(w, \nu)\right\| \leq L(\delta, \mu), \quad(w, \nu) \in \mathcal{M}_{\delta} \times \mathcal{B}_{\mu}^{s} .
$$


HC4 $\left\|\partial_{\theta} f_{3}\right\| \leq K_{\theta}$.

Similarly as before we call $K_{\omega}=\|D \omega\|_{0}, K_{l}=\max \left(\left\|D A_{-}\right\|_{0},\left\|D A_{+}\right\|_{0},\|D B\|_{0}\right)$ and $K_{f}=\left\|D f_{3}\right\|_{0}$.

Formula (3.1) also applies to $g$, already defined in (2.2). In that case we shall write $g(w, \nu)=\varphi_{g 1}(w, \nu) x+\varphi_{g 2}(w, \nu) v$. Note that if $\gamma \in \Sigma_{c, M}$ with $c \in(0,1]$

$$
\left\|\partial_{s} f_{j}(z, \gamma(z))\right\| \leq L, \quad s=x, v, \quad\left\|\partial_{\theta} f_{j}(z, \gamma(z))\right\| \leq K_{m}\|x\|, \quad j=1,2,4,
$$

and in particular $\left\|\partial_{s} g(z, \gamma(z))\right\| \leq L, s=x, v$, and $\left\|\partial_{\theta} g(z, \gamma(z))\right\| \leq K_{m}\|x\|$.

Theorem 3.1 Let $F_{\nu}: \mathcal{M}_{\delta} \rightarrow \mathcal{M}$ satisfy hypotheses HC1 to HC4. Then there exist $\delta_{k}$ and $\mu_{k}$ such that, if $0<\delta<\delta_{k}$ and $\|\nu\|<\mu_{k}$, the function $\gamma_{\nu}^{s}(x, \theta)$ given by Theorem 2.1 whose graph is the stable invariant manifold of $\mathbb{T}_{0}$ is $C^{k}$. Moreover if we ask

HC5 For $j=1,2,4, \varphi_{j i}$ is $C^{r}$ with respect to $(\theta, \nu),\left\|\partial_{\nu} f_{j}(w, \nu)\right\| \leq K_{m}\|(x, y, r)\|$ and $\left\|\partial_{\nu} f_{3}\right\| \leq K_{\theta}$

then $\gamma^{s}$ is $C^{k}$ with respect to $(x, \theta, \nu)$.

Remark 3.2 Like in the Lipschitz case, we shall only prove the results for the stable manifold. The ones for the unstable manifold, when $F$ is invertible, follow by considering $F^{-1}$.

Remark 3.3 In fact it follows from the proof that we obtain $\gamma^{s}$ such that $\partial_{\theta}^{k} \gamma^{s}$ factorizes as $\partial_{\theta}^{k} \gamma^{s}(x, \theta)=\psi_{k}(x, \theta) x$ with $\psi_{k}$ continuous. Integrating $k$-times with respect to $\theta$ we have that $\gamma^{s}(x, \theta)=\tilde{\gamma}^{s}(x, \theta) x$ with $\tilde{\gamma}^{s}$ being $C^{k}$ with respect to $\theta$. Notice that if $p>1$ this factorization may be not unique.

Remark 3.4 As in the Lipschitz case we can let $A_{ \pm}$and $B$ depend on $\nu$ provided that HC1 and HC2 hold uniformly in $\nu$. See Remark 2.3.

Remark 3.5 Under hypotheses HC1-HC4 the values $\mu_{k}$ and $\delta_{k}$ given by Theorem 3.1 depend, in principle, on $k$. In the proof of the Theorem it is necessary to take $\mu_{k}$ and $\delta_{k}$ smaller each time the degree of differentiability is increased. However, although nothing can be done (without changing the hypotheses) about the decreasing of $\mu_{k}$, if $F$ is invertible on the stable manifold, the value of $\delta_{k}$ can be restored to its original size. More concretely, if we are under hypotheses of type $C^{k}$, we obtain a function $\gamma(x, \theta)$ defined in $\mathcal{B}_{\delta_{k}}^{m} \times \mathbb{T}^{n}$, which can be extended by iteration by $F^{-1}$. But since the hypotheses in the $C^{k}$ case imply those of the Lipschitz case, in fact there is a Lipschitz solution defined in $\mathcal{B}_{\delta_{k}}^{m} \times \mathbb{T}^{n}$. By Remark 2.4, the extension (which is $C^{k}$ ) and the Lipschitz manifold, must coincide. Therefore the $C^{k}$ invariant manifold is defined in a neighborhood of $\mathbb{T}_{0}$ whose size does not depend on $k$.

\section{The Fiber Contraction Theorem}

Although it is quite well known, in this section we recall the fiber contraction theorem since we shall use it several times. Also we describe how we shall apply it, in order to do not repeat the same arguments several times. 
Theorem 4.1 Let $\Sigma$ and $D \Sigma$ be metric spaces, $D \Sigma$ complete, and $\Gamma: \Sigma \times D \Sigma \rightarrow \Sigma \times D \Sigma a$ map of the form $\Gamma(\gamma, \psi)=(G(\gamma), H(\gamma, \psi))$. Assume that

(1) $G: \Sigma \rightarrow \Sigma$ has an attracting fixed point $\gamma_{\infty} \in \Sigma$ (i.e. $\lim _{k \rightarrow \infty} G^{k}(\gamma)=\gamma_{\infty}, \forall \gamma \in \Sigma$ ).

(2) $\lim \sup _{n \rightarrow \infty} \operatorname{Lip} H\left(G^{n}(\gamma),.\right)<1$ for each $\gamma \in \Sigma$.

Let $\psi_{\infty} \in D \Sigma$ be a fixed point for $H\left(\gamma_{\infty},.\right)$.

(3) $H$ is continuous with respect to $\gamma$ at $\left(\gamma_{\infty}, \psi_{\infty}\right)$.

Then $\left(\gamma_{\infty}, \psi_{\infty}\right)$ is an attracting fixed point of $\Gamma$.

Remark 4.2 We have not asked $\Sigma$ to be complete, instead we assume that G already has an attracting fixed point.

The statement is a slight modified version of the one in [12]. Its proof is completely analogous.

One way of using this theorem is to deal with operators $\Gamma=(G, F)$ defined in suitable spaces of $C^{k-1}$ functions such that if $\gamma$ is $C^{k}, G(\gamma)$ is $C^{k}$ and if $\psi=D \gamma$

$$
D(G(\gamma))=H(\gamma, \psi)
$$

Then let $\left(\gamma_{j}, \psi_{j}\right)=\Gamma^{j}\left(\gamma_{0}, \psi_{0}\right)$. Applying (4.1) inductively we have that $\psi_{j}=D \gamma_{j}$ for all $j$.

If we take a function $\gamma_{0}$ of class $C^{k}$ and $\psi_{0}=D \gamma_{0}$, the iterates $\gamma_{j}=G^{j}\left(\gamma_{0}\right)$ are $C^{k}$ and converge in $C^{k-1}$. Also $\left(\psi_{j}\right)$ converges in $C^{k-1}$. Then $\left(D \gamma_{j}\right)$ converges in $C^{k-1}$ and therefore $\gamma_{\infty}$ is $C^{k}$.

Another possibility is to take $\Sigma$ as an space of $C^{k-1}$ functions and $D \Sigma$ a suitable space of $C^{0}$ functions and the operator $\Gamma$ such that if $\gamma$ is $C^{k}, G(\gamma)$ is $C^{k}$ and if $\psi=D^{k} \gamma$

$$
D^{k}(G(\gamma))=H(\gamma, \psi) .
$$

Taking $\gamma_{0}$ of class $C^{k}$, and $\psi_{0}=D^{k} \gamma_{0}$, the iterates $\gamma_{j}=G^{j}\left(\gamma_{0}\right)$ are $C^{k}$ and converge in $C^{k-1}$. Also $\left(\psi_{j}\right)$ converges in $C^{0}$ and then $\left(D^{k} \gamma_{j}\right)$ converges in $C^{0}$ and therefore $\gamma_{\infty}$ is $C^{k}$.

\section{$5 \quad$ Proof of Theorem 3.1}

Proof of the $C^{1}$ case. We will first prove the $C^{1}$ case and then we will proceed by induction. The $C^{1}$ hypotheses imply the Lipschitz ones; therefore if $c \in(0,1]$ and $M$ verifies $(2.18)$ the map $G$ defined by formula $(2.5)$ is a contraction in the space $\Sigma_{c, M}$ defined in (2.7). Let $\gamma^{s}$ be its unique fixed point in $\Sigma_{c, M}$. It is an attracting fixed point of $G$. We want to see that $\gamma^{s}$ is $C^{1}$. We consider the set

$$
\tilde{\Sigma}=\{\gamma \in \Sigma ; \gamma(z)=\tilde{\gamma}(z) x, \tilde{\gamma} \text { continuous }\}
$$

and $\tilde{\Sigma}_{c, M}=\Sigma_{c, M} \cap \tilde{\Sigma}$. If $\gamma \in \tilde{\Sigma}$, the representation $\gamma(z)=\tilde{\gamma}(z) x$ is not unique. However from any representation we can get a sort of canonical one, $\gamma(z)=\tilde{\gamma}^{c}(z) x$, with

$$
\tilde{\gamma}_{j}^{c}(z)=\tilde{\gamma}_{j}(0, \theta)+x_{j}<\tilde{\gamma}(x, \theta)-\tilde{\gamma}(0, \theta), x>/\|x\|^{2}
$$

which is continuous. Using this form one readily sees that $\tilde{\Sigma}$ is complete.

We have that $G$ sends $\tilde{\Sigma}_{c, M}$ to $\tilde{\Sigma}_{c, M}$. Indeed, by (3.1) applied to $g$

$$
G(\gamma)(z)=C(\theta)^{-1}\left[\varphi_{g 1}(z, \gamma(z)) x+\varphi_{g 2}(z, \gamma(z)) \tilde{\gamma}(z) x+\tilde{\gamma}(h(\gamma)(z)) h_{1}(\gamma(z))\right]
$$


and using that $h_{1}(\gamma)(z)=A(\theta) x+\varphi_{11}(z, \gamma(z)) x+\varphi_{12}(z, \gamma(z)) \gamma(z)$ we get $G(\gamma)(z)=\tilde{\gamma}^{1}(z) x$ with $\tilde{\gamma}^{1}$ continuous. Since $\tilde{\Sigma}$ is closed, the fixed point of $G$ in $\Sigma_{c, M}$ belongs in fact to $\tilde{\Sigma}_{c, M}$.

To apply the Fiber Contraction Theorem we look for $H$ such that $D[G(\gamma)]=H(\gamma, D \gamma)$. For that we derivate formally $G(\gamma)$ and we substitute $\partial_{x} \gamma, \partial_{\theta} \gamma$ by $\psi_{0}$ and $\psi_{1}$. In this way we have the map $H(\gamma, \psi)=\left(H_{0}(\gamma, \psi), H_{1}(\gamma, \psi)\right)$ defined by

$$
\begin{aligned}
H_{0}(\gamma, \psi)(z)= & C^{-1}(\theta)\left(\partial_{x} g(z, \gamma(z))+\partial_{v} g(z, \gamma(z)) \psi_{0}(z)\right. \\
& +\psi_{0}(h(z))\left(A(\theta)+\partial_{x} f_{0}(z, \gamma(z))+\partial_{v} f_{0}(z, \gamma(z)) \psi_{0}(z)\right) \\
& +\psi_{1}(h(z))\left(\partial_{x} \omega(x, \gamma(z))+\partial_{v} \omega(x, \gamma(z)) \psi_{0}(z)+\partial_{x} f_{3}(z, \gamma(z))\right. \\
& \left.\left.+\partial_{v} f_{3}(z, \gamma(z)) \psi_{0}(z)\right)\right), \\
H_{1}(\gamma, \psi)(z)= & \partial_{\theta} C^{-1}(\theta)(g(z, \gamma(z))+\gamma(h(z)))+C^{-1}(\theta)\left(\partial_{\theta} g(z, \gamma(z))+\partial_{v} g(z, \gamma(z)) \psi_{1}(z)\right. \\
& +\psi_{0}(h(z))\left(\partial_{\theta} A(\theta) x+\partial_{\theta} f_{1}(z, \gamma(z))+\partial_{v} f_{1}(z, \gamma(z)) \psi_{1}(z)\right) \\
& \left.+\psi_{1}(h(z))\left(I+\partial_{v} \omega(x, \gamma(z)) \psi_{1}(z)+\partial_{\theta} f_{3}(z, \gamma(z))+\partial_{v} f_{3}(z, \gamma(z)) \psi_{1}(z)\right)\right) .
\end{aligned}
$$

Let $E=C^{0}\left(\mathcal{B}_{\delta}^{m} \times \mathbb{T}^{n}, L\left(\mathbb{R}^{m} \times \mathbb{R}^{n}, \mathbb{R}^{p} \times \mathbb{R}^{q}\right)\right)$. We introduce the Banach space

$$
D \Sigma=\left\{\psi=\left(\psi_{0}, \psi_{1}\right) \in E ; \psi_{1}(z)=\tilde{\psi}_{1}(z) x, \tilde{\psi}_{1} \text { continuous, }\left\|\psi_{0}\right\|_{0}<\infty,\left\|\psi_{1}\right\|_{1}<\infty\right\}
$$

with the norm

$$
\|\psi\|_{D \Sigma}=\alpha_{0}\left\|\psi_{0}\right\|_{0}+\alpha_{1}\left\|\psi_{1}\right\|_{1}
$$

where $\alpha_{0}$ and $\alpha_{1}$ are suitable positive constants and

$$
D \Sigma_{c, M}=\left\{\psi \in D \Sigma ;\left\|\psi_{0}\right\|_{0} \leq c,\left\|\psi_{1}\right\|_{1} \leq M\right\}
$$

Lemma 5.1 If $\delta$ and $\mu$ are small enough, for any $\gamma \in \tilde{\Sigma}_{c, M}$ and $\psi \in D \Sigma_{c, M}, H(\gamma, \psi) \in$ $D \Sigma_{c, M}$.

Proof of Lemma 5.1. The condition $H_{1}(\gamma, \psi)(z)=\tilde{\gamma}^{1}(z) x$ with $\tilde{\gamma}^{1}$ continuous holds because the term $g(z, \gamma(z))+\gamma(h(z))$ factorizes $x$, because $\psi_{1}(h(z))=\tilde{\psi}_{1}(h(z)) h_{1}(z)$, and also because $\partial_{\theta} f_{j}(z, \gamma(z))=\partial_{\theta} \varphi_{1 j}(z, \gamma(z)) x+\partial_{\theta} \varphi_{2 j}(z, \gamma(z)) \gamma(z)$ obtained from HC3 by derivation with respect to $\theta$. Moreover

$$
\begin{aligned}
\left\|H_{0}(\gamma, \psi)(z)\right\| \leq & \beta\left(L+L c+c\left(\lambda^{-1}+L+L c\right)\right. \\
& \left.+M\left(\lambda^{-1}+L\right)\|x\|\left(K_{\omega}+K_{\omega} c+K_{f}+c K_{f}\right)\right) \\
\leq & c \beta \lambda^{-1}+\beta L(1+c)^{2}+\beta M \delta\left(K_{\omega}+K_{f}\right)(1+c)
\end{aligned}
$$

which is smaller than $c$, since $\beta \lambda^{-1}<1$, if $\delta$ and $\mu$ are small enough; and

$$
\begin{aligned}
\left\|H_{1}(\gamma, \psi)(z)\right\| \leq & K_{l} \beta^{2}\left(L+\left(\lambda^{-1}+L\right) c\right)\|x\|+\beta\left(\left(K_{m}+L M\right)\|x\|\right. \\
& +c\left(K_{l}+K_{m}+L M\right)\|x\| \\
& \left.+M\left(\lambda^{-1}+L\right)\|x\|\left(1+K_{\omega} M\|x\|+K_{\theta}+K_{f} M\|x\|\right)\right) \\
\leq \quad & \left(K_{l} \beta^{2} \lambda^{-1} c+\beta c\left(K_{l}+K_{m}\right)+\beta M \lambda^{-1}\left(1+K_{\theta}\right)+\beta K_{m}+O(L)+O(\delta)\right)\|x\|
\end{aligned}
$$

which proves the Lemma because $M$ satisfies (2.18). 
Lemma 5.2 If $\alpha_{0}>\frac{\beta\left(K_{l}+K_{m}\right) \alpha_{1}}{1-\beta \lambda^{-1}}$, the map $H(\gamma,$.$) is a contraction, uniform with respect to$ $\gamma$.

Proof of Lemma 5.2. Decomposing the differences in a suitable telescopic form

$$
\begin{aligned}
& \left\|H_{0}(\gamma, \psi)(z)-H_{0}\left(\gamma, \psi^{\prime}\right)(z)\right\| \\
& \leq \quad \beta\left(L+c L+\left(K_{\omega}+K_{f}\right) M\left(\lambda^{-1}+L\right)\|x\|\right)\left\|\psi_{0}(z)-\psi_{0}^{\prime}(z)\right\| \\
& \quad+\beta\left(\lambda^{-1}+L+c L\right)\left\|\psi_{0}(h(z))-\psi_{0}^{\prime}(h(z))\right\| \\
& \quad+\beta\left(K_{\omega}+K_{f}\right)(1+c)\left\|\psi_{1}(h(z))-\psi_{1}^{\prime}(h(z))\right\|,
\end{aligned}
$$

and

$$
\begin{aligned}
& \left\|H_{1}(\gamma, \psi)(z)-H_{1}\left(\gamma, \psi^{\prime}\right)(z)\right\| \\
& \leq \quad \beta\left(L+c L+M\left(\lambda^{-1}+L\right)\|x\|\left(K_{\omega}+K_{f}\right)\right)\left\|\psi_{1}(z)-\psi_{1}^{\prime}(z)\right\| \\
& \quad+\beta\left(K_{l}+K_{m}+L M\right)\|x\|\left\|\psi_{0}(h(z))-\psi_{0}^{\prime}(h(z))\right\| \\
& \quad+\beta\left(1+K_{\theta}+M\|x\|\left(K_{\omega}+K_{f}\right)\right)\left\|\psi_{1}(h(z))-\psi_{1}^{\prime}(h(z))\right\| .
\end{aligned}
$$

Therefore,

$$
\begin{aligned}
\alpha_{0}\left\|H_{0}(\gamma, \psi)-H_{0}\left(\gamma, \psi^{\prime}\right)\right\|_{0}+\alpha_{1}\left\|H_{1}(\gamma, \psi)-H_{1}\left(\gamma, \psi^{\prime}\right)\right\|_{1} \\
\leq \quad \beta\left(\alpha_{0} \lambda^{-1}+\alpha_{1}\left(K_{l}+K_{m}\right)+O(L)+O(\delta)\right)\left\|\psi_{0}-\psi_{0}^{\prime}\right\|_{0} \\
+\beta\left(\alpha_{1}\left(1+K_{\theta}\right) \lambda^{-1}+O(L)+O(\delta)\right)\left\|\psi_{1}-\psi_{1}^{\prime}\right\|_{1} .
\end{aligned}
$$

Therefore, choosing suitable $\alpha_{0}, \alpha_{1}$, for instance $\alpha_{1}=1$ and $\alpha_{0}>\beta \alpha_{1}\left(K_{l}+K_{m}\right) /\left(1-\beta \lambda^{-1}\right)$, and taking $\delta, \mu$ small enough we have that $H(\gamma,$.$) is a contraction.$

We have that $\gamma \mapsto H(\gamma, \psi)$ is continuous. To prove it we have to see that $\alpha_{0}|| H_{0}\left(\gamma^{\prime}, \psi\right)-$ $H_{0}(\gamma, \psi)\left\|_{0}+\alpha_{1}\right\| H_{1}\left(\gamma^{\prime}, \psi\right)-H_{1}(\gamma, \psi) \|_{1}$ is small if $\left\|\gamma^{\prime}-\gamma\right\|_{1}$ is small. Decomposing the difference in telescopic form, the more delicate term to control is the one which contains $\left\|\psi_{1}\left(h\left(\gamma^{\prime}\right)\right)-\psi_{1}(h(\gamma))\right\|_{1}$. We have

$$
\begin{aligned}
&\|x\|^{-1}\left\|\tilde{\psi}_{1}\left(h\left(\gamma^{\prime}\right)(z)\right)\left(A(\theta) x+f_{1}\left(z, \gamma^{\prime}(z)\right)\right)-\tilde{\psi}_{1}(h(\gamma)(z))\left(A(\theta) x+f_{1}(z, \gamma(z))\right)\right\| \\
& \leq\|x\|^{-1}\left\|\left[\tilde{\psi}_{1}\left(h\left(\gamma^{\prime}\right)(z)\right)-\tilde{\psi}_{1}(h(\gamma)(z))\right] A(\theta) x\right\| \\
&+\left\|\tilde{\psi}_{1}\left(h\left(\gamma^{\prime}\right)(z)\right)-\tilde{\psi}_{1}(h(\gamma)(z))\right\|\|x\|^{-1}\left\|f_{1}\left(z, \gamma^{\prime}(z)\right)\right\| \\
&+\| \tilde{\psi}_{1}\left(h(\gamma)(z)\|\| x\left\|^{-1}\right\| f_{1}\left(z, \gamma^{\prime}(z)\right)-f_{1}(z, \gamma(z)) \|\right.
\end{aligned}
$$

which is small by the uniform continuity of $\tilde{\psi}_{1}$, because $\|x\|^{-1}\left\|f_{1}\left(z, \gamma^{\prime}(z)\right)\right\|$ is bounded by (2.9) and $\|x\|^{-1}\left\|f_{1}\left(z, \gamma^{\prime}(z)\right)-f_{1}(z, \gamma(z))\right\| \leq\|x\|^{-1} L\left\|\gamma^{\prime}(z)-\gamma(z)\right\| \leq L\left\|\gamma^{\prime}-\gamma\right\|_{1}$. Remember that $\gamma^{s}$ is an attracting fixed point of the map $G: \tilde{\Sigma}_{c, M} \rightarrow \tilde{\Sigma}_{c, M}$. The Fiber Contraction Theorem implies that $\Gamma=(G, H): \tilde{\Sigma}_{c, M} \times D \Sigma_{c, M} \longrightarrow \tilde{\Sigma}_{c, M} \times D \Sigma_{c, M}$ has a (unique) attracting fixed point. Finally, arguing as is indicated in Section 4, for the first situation described, taking, for instance, $\gamma_{0}=0, \psi_{0}=0$, as initial functions for the iteration, we get that $\gamma^{s}$ is $C^{1}$.

Proof of the $C^{k}$ case

For $i \geq 2$, given $\hat{c}=\left(c_{1}, \ldots, c_{i-1}\right)$ and $\hat{M}=\left(M_{1}, \ldots, M_{i-1}\right)$ with $c_{1}=c$ and $M_{1}=M$ we consider the spaces

$$
\Sigma^{i}=\left\{\gamma \in C^{i-1} ; \gamma(0, \theta)=0,\left\|D^{j} \gamma\right\|_{0}<\infty,\left\|\partial_{\theta}^{j} \gamma\right\|_{1}<\infty, 1 \leq j \leq i-1\right\}
$$


with the norm

$$
\|\gamma\|_{\Sigma}=\max _{1 \leq j \leq i-1}\left(\left\|D^{j} \gamma\right\|_{0},\left\|\partial_{\theta}^{j} \gamma\right\|_{1}\right)
$$

and

$$
\sum_{\hat{c}, \hat{M}}^{i}=\left\{\gamma \in \Sigma^{i} ;\left\|D^{j} \gamma\right\|_{\alpha} \leq c_{j},\left\|\partial_{\theta}^{j} \gamma\right\|_{1} \leq M_{j}, 0 \leq j \leq i-1\right\}
$$

where we take $\left\|D^{j} \gamma\right\|_{\alpha}=\sum_{l=0}^{j-1} \alpha_{l}\left\|\partial_{\theta}^{l} \partial_{x}^{j-l} \gamma\right\|_{0}$ and $\alpha_{l}$ are positive numbers (to be fixed later on). Also

$$
\begin{aligned}
D \Sigma^{i}=\{\psi= & \left(\psi_{0}, \ldots, \psi_{i}\right) ; \psi_{j} \in C^{0}\left(\mathcal{B}_{\delta} \times \mathbb{T}^{n}, L^{i-j}\left(\mathbb{R}^{m}, L^{j}\left(\mathbb{R}^{n}, \mathbb{R}^{p+q}\right)\right)\right), \\
& \left.\psi_{i}(z)=\tilde{\psi}_{i}(z) x, \text { with } \tilde{\psi}_{i} \in C^{0},\left\|\psi_{j}\right\|_{0}<\infty, 0 \leq j \leq i-1,\left\|\psi_{i}\right\|_{1}<\infty\right\}
\end{aligned}
$$

with the norm

$$
\|\psi\|_{D \Sigma}=\alpha_{0}\left\|\psi_{0}\right\|_{0}+\cdots+\alpha_{i-1}\left\|\psi_{i-1}\right\|_{0}+\eta\left\|\psi_{i}\right\|_{1}
$$

where $\eta$ is a constant and

$$
D \Sigma_{c_{i}, M_{i}}^{i}=\left\{\psi \in D \Sigma^{i} ; \alpha_{0}\left\|\psi_{0}\right\|_{0}+\cdots+\alpha_{i-1}\left\|\psi_{i-1}\right\|_{0} \leq c_{i},\left\|\psi_{i}\right\|_{1} \leq M_{i}\right\}
$$

and we consider the operator $G: \Sigma_{\hat{c}, \hat{M}}^{i} \rightarrow \Sigma_{\hat{c}, \hat{M}}^{i}$ defined by $(2.5)$. We define $H(\gamma, \psi)$ from the i-derivative of $G(\gamma)$. We define $H_{j}(\gamma, \psi)$ as the derivative $\partial_{\theta}^{j} \partial_{x}^{i-j}$ of $G(\gamma)$ when we substitute $\partial_{\theta}^{j} \partial_{x}^{i-j} \gamma$ by $\psi_{j}, j=0, \ldots, i$, in order to have $D^{i-1}[G(\gamma)]=H\left(\gamma, D^{i-1} \gamma\right)$. We have $H: \Sigma_{\hat{c}, \hat{M}}^{i} \times D \Sigma_{c_{i}, M_{i}}^{i} \rightarrow D \Sigma_{c_{i}, M_{i}}^{i}$ (In fact the operator $H$ depends on $i$. However we use the same symbol in order to do not complicate the notation too much.)

In this way, denoting $h(\gamma)$ by $h$ and $(z, \gamma(z))=(I, \gamma)(z)$, we have that

$$
\begin{aligned}
H_{j}(\gamma, \psi)= & C^{-1}\left(\partial_{\theta}^{j} \partial_{x}^{i-j} g \circ(I, \gamma)+\cdots+\partial_{v} g \circ(I, \gamma) \psi_{j}+\sum_{l=0}^{i}\left(\psi_{l} \circ h\right) T_{l, j}\right. \\
& \left.+\left(\partial_{x} \gamma \circ h\right) \partial_{\theta}^{j} \partial_{x}^{i-j} h_{1}+\left(\partial_{\theta} \gamma \circ h\right) \partial_{\theta}^{j} \partial_{x}^{i-j} h_{2}\right)+\cdots
\end{aligned}
$$

where dots stand for terms which depend on $\gamma$ and its derivatives of order less or equal than $i-1, T_{l, j}$ is a sum of terms each one being a product of derivatives of $h_{1}$ and $h_{2}$. It contains $j$ derivatives with respect to $\theta$ and $i-j$ derivatives with respect to $x$, and from those there are $l$ derivatives of $h_{2}$ and $i-l$ derivatives of $h_{1}$. In particular $T_{0, j}=\left(\partial_{\theta} h_{1}\right)^{j}\left(\partial_{x} h_{1}\right)^{i-j}$ and $T_{i, j}=\left(\partial_{\theta} h_{2}\right)^{j}\left(\partial_{x} h_{2}\right)^{i-j}$.

Furthermore $H_{j}$ also depends on $\psi$ through the derivatives of $h$ :

$$
\begin{aligned}
\partial_{\theta}^{j} \partial_{x}^{i-j}\left[h_{1}(\gamma)\right](z)= & \partial_{\theta}^{j} \partial_{x}^{i-j} f_{1}(z, \gamma(z))+\cdots+\partial_{v} f_{1}(z, \gamma(z)) \psi_{j}(z), \quad 0 \leq j \leq i-2, \\
\partial_{\theta}^{i-1} \partial_{x}\left[h_{1}(\gamma)\right](z)= & \partial_{\theta}^{i-1} A(\theta)+\partial_{\theta}^{i-1} \partial_{x} f_{1}(z, \gamma(z))+\cdots+\partial_{v} f_{1}(z, \gamma(z)) \psi_{i-1}(z), \\
\partial_{\theta}^{i}\left[h_{1}(\gamma)\right](z)= & \partial_{\theta}^{i} A(\theta) x+\partial_{\theta}^{i} f_{1}(z, \gamma(z))+\cdots+\partial_{v} f_{1}(z, \gamma(z)) \psi_{i}(z), \\
\partial_{x}^{i}\left[h_{2}(\gamma)\right](z)= & \partial_{x}^{i} \omega(x, \gamma(z))+\cdots+\partial_{v} \omega(x, \gamma(z)) \psi_{0}(z) \\
& +\partial_{x}^{i} f_{3}(z, \gamma(z))+\cdots+\partial_{v} f_{3}(z, \gamma(z)) \psi_{0}(z), \\
\partial_{\theta}^{j} \partial_{x}^{i-j}\left[h_{2}(\gamma)\right](z)= & \partial_{v} \omega(x, \gamma(z)) \psi_{j}(z)+\cdots \\
& +\partial_{\theta}^{j} \partial_{x}^{i-j} f_{3}(z, \gamma(z))+\cdots+\partial_{v} f_{3}(z, \gamma(z)) \psi_{j}(z), \quad 1 \leq j \leq i .
\end{aligned}
$$


Hence $H_{j}(\gamma, \psi)$ has the form

$$
\begin{aligned}
& C^{-1}\left[\partial_{v} g \circ(I, \gamma)+\left(\partial_{x} \gamma \circ h\right) \partial_{v} f_{1} \circ(I, \gamma)+\left(\partial_{\theta} \gamma \circ h\right)\left(\partial_{v} \omega \circ(I, \gamma)+\partial_{v} f_{3} \circ(I, \gamma)\right)\right] \psi_{j} \\
& +C^{-1} \sum_{l=0}^{i}\left(\psi_{l} \circ h\right) T_{l, j}+\tilde{H}_{j}(\gamma) .
\end{aligned}
$$

We will prove by induction that if $2 \leq i \leq k$, given $\hat{c}=\left(c_{1}, \ldots, c_{i-1}\right)$ and $\hat{M}=$ $\left(M_{1}, \ldots, M_{i-1}\right)$, there exist $c_{i}$ and $M_{i}$ such that

(a) $G: \Sigma_{\hat{c}, \hat{M}}^{i} \longrightarrow \Sigma_{\hat{c}, \hat{M}}^{i}$ is well defined and has an attracting fixed point $\gamma_{\infty}$,

(b) $H: \sum_{\hat{c}, M^{i}}^{i} \times D \Sigma_{c_{i}, M_{i}}^{i} \longrightarrow D \Sigma_{c_{i}, M_{i}}^{i}$ is well defined,

(c) $H(., \psi): \Sigma_{\hat{c}, \hat{M}}^{i} \longrightarrow D \Sigma_{c_{i}, M_{i}}^{i}$ is continuous,

(d) $H(\gamma,):. D \sum_{c_{i}, M_{i}}^{i} \longrightarrow D \sum_{c_{i}, M_{i}}^{i}$ is a contraction.

We first observe that $\Sigma_{\hat{c}, \hat{M}}^{i} \subset \tilde{\Sigma}_{c_{1}, M_{1}}$. If $\gamma \in \Sigma_{\hat{c}, \hat{M}}^{i}$

$$
\begin{array}{ll}
\left\|\partial_{x} h_{1}(\gamma)(z)\right\| \leq N_{1}, \quad & \left\|\partial_{x} h_{2}(\gamma)(z)\right\| \leq N_{2}, \\
\left\|\partial_{\theta} h_{1}(\gamma)(z)\right\| \leq N_{2}\|x\|, & \left\|\partial_{\theta} h_{2}(\gamma)(z)\right\| \leq N_{3},
\end{array}
$$

where, to simplify the notation, we have introduced the constants $N_{1}=\lambda^{-1}+L+L c_{1}$, $N_{2}=\max \left(\left(K_{\omega}+K_{f}\right)\left(1+c_{1}\right), K_{l}+K_{m}+L M_{1}\right)$ and $N_{3}=1+K_{\theta}+\left(K_{\omega}+K_{f}\right) M_{1} \delta$. Notice that $N_{1}$ and $N_{3}$ are very close to $\lambda^{-1}$ and $1+K_{\theta}$ respectively. To bound $T_{l, j}$ we observe that if $l<j$ its terms must contain at least one derivative $\partial_{\theta} h_{1}(\gamma)$ as a factor, which is bounded by $N_{2}\|x\|$. On the other hand $T_{j, j}$ has one term $\left(\partial_{\theta} h_{2}\right)^{j}\left(\partial_{x} h_{1}\right)^{i-j}$ and all the others have at least one factor $\partial_{\theta} h_{1}$. Then there exist a constant $N_{5}$ such that

$$
\begin{aligned}
T_{l, j} \leq N_{5} / \beta, & l>j, \\
T_{j, j} \leq N_{3}^{j} N_{1}^{i-j}+\left(N_{5} / \beta\right)\|x\|, & \\
T_{l, j} \leq\left(N_{5} / \beta\right)\|x\|, & l<j,
\end{aligned}
$$

and we take a value for $N_{5}$ to be valid for $i \leq k$. Let $\Lambda, \eta$ be positive numbers such that

$$
\beta \lambda^{-1}\left(1+K_{\theta}\right)^{i}<\Lambda<1, \quad \eta \leq 1, \quad \Lambda+2 N_{5} \eta<1 .
$$

We take $\alpha_{0}=1$ and we choose $\alpha_{l}$ inductively so that

$$
\alpha_{l}=\left(\alpha_{0}+\cdots+\alpha_{l-1}\right) \eta^{-1}, \quad 1 \leq l \leq i-1 .
$$

Lemma 5.3 Assuming (a) - (d) for $i-1$ we have that (a) holds for $i, i \leq k$.

Proof. Assume that $\gamma \in \sum_{\hat{c}, \hat{M}}^{i} \subset \sum_{\hat{c}, \hat{M}}^{i-1}$. From (a) we already know that $G(\gamma) \in \Sigma_{\hat{c}, \hat{M}^{\circ}}^{i-1}$. We have to check that $G(\gamma) \in \Sigma_{\hat{c}, \hat{M}}^{i}$. The conditions $\left\|D^{i-1} \gamma\right\|_{\alpha} \leq c_{i-1}$ and $\left\|\partial_{\theta}^{i-1} \gamma\right\|_{1} \leq M_{i-1}$ imply that $D^{i-1} \gamma \in D \Sigma_{c_{i-1}, M_{i-1}}^{i-1}$. Moreover the conditions $D^{i-1}[G(\gamma)]=H\left(\gamma, D^{i-1} \gamma\right)$ and 
(b) mean that $D^{i-1}[G(\gamma)] \in D \Sigma_{c_{i-1}, M_{i-1}}^{i-1}$ so that $G(\gamma) \in \Sigma_{\hat{c}, \hat{M}^{i}}^{i}$. When $i=2$ we must start from $G: \tilde{\Sigma}_{c_{1}, M_{1}} \longrightarrow \tilde{\Sigma}_{c_{1}, M_{1}}$.

We apply the Fiber Contraction Theorem to $\Gamma=(G, H): \sum_{\hat{c}, \hat{M}}^{i-1} \times D \Sigma_{c_{i-1}, M_{i-1}}^{i-1} \longrightarrow$ $\sum_{\hat{c}, \hat{M}}^{i-1} \times D \sum_{c_{i-1}, M_{i-1}}^{i-1}$ as it is described in Section 4 and we get that if $\gamma \in \sum_{\hat{c}, \hat{M}}^{i}$, the sequence of iterates $G^{j}(\gamma)$ converges to a $C^{i}$-function in $\Sigma_{\hat{c}, \hat{M}^{i}}^{i}$. Therefore $G$ has an attracting fixed point.

Lemma 5.4 Given $\hat{c}=\left(c_{1}, \ldots, c_{i-1}\right), M_{i-1}$, there exist $c_{i}, M_{i}, \mu_{0}$ and $\delta_{0}$, positive constants, such that for all $\nu \in \mathcal{B}_{\mu_{0}}^{s}, 0<\delta<\delta_{0}$ and for any $\gamma \in \Sigma_{\hat{c}, \hat{M}}^{i}$ and $\psi \in D \Sigma_{c_{i}, M_{i}}^{i}, H(\gamma, \psi) \in$ $D \sum_{c_{i}, M_{i}}^{i} \cdot$

Proof of Lemma 5.4. It is clear from the definition of $H_{j}(\gamma, \psi)$ that all its terms which are independent of $\psi$ can be collected together and their norm can be bounded by some constant $N_{4}$ (which, of course, depends on $i$ but can be chosen independently on $j$ ). In this way

$$
\begin{aligned}
& \left\|H_{j}(\gamma, \psi)(z)\right\| \leq N_{4}+\beta L\left\|\psi_{j}\right\|+\beta c_{1} L\left\|\psi_{j}\right\|+\beta M_{1}\left(K_{\omega}+K_{f}\right)\left\|\psi_{j}\right\|\left(\lambda^{-1}+L\right)\|x\| \\
& \quad+\quad N_{5}\|x\|\left(\left\|\psi_{0}\right\|+\ldots+\left\|\psi_{j}\right\|\right)+\beta N_{3}^{j} N_{1}^{i-j}\left\|\psi_{j}\right\|+N_{5}\left(\left\|\psi_{j+1}\right\|+\ldots+\left\|\psi_{i}\right\|\right) \\
& \quad \leq \quad N_{4}+N_{5}\|x\|\left(\left\|\psi_{0}\right\|+\ldots+\left\|\psi_{j}\right\|\right)+\Lambda\left\|\psi_{j}\right\|+N_{5}\left(\left\|\psi_{j+1}\right\|+\ldots+\left\|\psi_{i}\right\|\right)
\end{aligned}
$$

if $\delta$ and $\mu$ small enough. We have

$$
\begin{aligned}
\alpha_{0} \| & H_{0}(\gamma, \psi)\left\|+\ldots+\alpha_{i-1}\right\| H_{i-1}(\gamma, \psi) \| \\
\leq & N_{4}\left(\alpha_{0}+\ldots+\alpha_{i-1}\right)+N_{5}\|x\|\left[\left(\alpha_{0}+\ldots+\alpha_{i-1}\right)\left\|\psi_{0}\right\|+\ldots+\alpha_{i-1}\left\|\psi_{i-1}\right\|\right] \\
& +\Lambda\left[\alpha_{0}\left\|\psi_{0}\right\|+\ldots+\alpha_{i-1}\left\|\psi_{i-1}\right\|\right] \\
& +N_{5}\left[\alpha_{0}\left\|\psi_{1}\right\|+\left(\alpha_{0}+\alpha_{1}\right)\left\|\psi_{2}\right\|+\ldots+\left(\alpha_{0}+\ldots+\alpha_{i-1}\right)\left\|\psi_{i}\right\|\right] .
\end{aligned}
$$

Using that $\left(\alpha_{0}+\ldots+\alpha_{l-1}\right)=\eta \alpha_{l}, 1 \leq l \leq i-1$, and $\left\|\psi_{i}\right\|_{0} \leq M_{i} \delta$

$$
\sum_{l=0}^{i-1} \alpha_{l}\left\|H_{l}\right\|_{0} \leq N_{4} \sum_{l=0}^{i-1} \alpha_{l}+O(\|x\|)+\Lambda c_{i}+N_{5} \eta c_{i}+\delta\left(N_{5} \sum_{l=0}^{i-1} \alpha_{l}\right) M_{i} .
$$

Now we check the condition for $H_{i}$. By its definition there are terms of the form 1) $\partial_{\theta}^{i}\left(C^{-1}(\theta)\right)[g(z, \gamma(z))+\gamma(h(\gamma)(z))]$. Since $g(0, \theta, \gamma(0, \theta))=0$ and $\gamma(h(\gamma)(0, \theta))=0$ there exists $C$ such that $\| \partial_{\theta}^{i}\left(C^{-1}(\theta)\right)[g(z, \gamma(z))+\gamma(h(\gamma(z))]\|\leq C\| x \|$. Up to the end of the proof $C$ will mean a suitable constant independent of $c_{i}$ and $M_{i}$.

2) Terms without $\psi_{j}$. There are two possibilities: either they come from the derivatives of $g(z, \gamma(z))$ or they come from the derivatives of $\gamma(h(\gamma)(z))$. In the first case, either they contain a term of the form $\partial_{\theta}^{l} \gamma(z), 1 \leq l \leq i-1$, which by definition of $\Sigma_{\hat{c}, \hat{M}}^{i}$, verifies $\left\|\partial_{\theta}^{l} \gamma(z)\right\| \leq M_{l}\|x\|$, or it is $\partial_{\theta}^{i} g(z, \gamma(z))$, which is also bounded by $C\|x\|$. In the second case, either they contain a term of the form $\partial_{\theta}^{l} h_{1}(\gamma)(z), l<i$, which is bounded by $C\|x\|$, or they contain a term with a factor $\partial_{\theta}^{j} \gamma(h(z)), j<i-1$, which also has the same kind of bound. 3) Terms including $\psi_{j}$. They are explicit in (5.3). Notice that for $0 \leq j \leq i-1, T_{j, i}$ which is of order $\|x\|$. 
Therefore, we have that

$$
\begin{aligned}
\left\|H_{i}(\gamma, \psi)(z)\right\| \leq & C\|x\|+\beta\left[L+c_{1} L+M_{1}\left(\lambda^{-1}+L\right)\|x\|\left(K_{\omega}+K_{f}\right)\right]\left\|\psi_{i}(z)\right\| \\
& +\beta \sum_{l=0}^{i-1}\left\|T_{l, i}\right\|\left\|\psi_{l} \circ h\right\|_{0}+\beta\left\|T_{i, i}\right\|\left\|\psi_{i} \circ h\right\|_{0} \\
\leq & C\|x\|+\beta\left[N_{3}^{i}\left(\lambda^{-1}+L\right)+O(L+\delta)\right] M_{i}\|x\|+N_{5}\|x\| c_{i} .
\end{aligned}
$$

Then, if $\delta$ and $\mu$ are sufficiently small $c_{i}$ and $M_{i}$ must verify

$$
\begin{aligned}
& \left(\Lambda+N_{5} \eta\right) c_{i}+\delta\left(N_{5} \sum_{l=0}^{i-1} \alpha_{l}\right) M_{i}+N_{4} \sum_{l=0}^{i-1} \alpha_{l}+O(\delta) \leq c_{i} \\
& N_{5} c_{i}+\Lambda M_{i}+O(\delta+L) \leq M_{i} .
\end{aligned}
$$

If $\delta$ is small there exists $c_{i}$ and $M_{i}$ satisfying (5.4) and (5.5), and hence $H(\gamma, \psi) \in D \Sigma_{c_{i}, M_{i}}^{i}$.

Lemma 5.5 The map $\gamma \mapsto H(\gamma, \psi)$ is continuous.

Proof. To prove that $H$ is continuous with respect to $\gamma$ we have to see that, with $\gamma \in \Sigma_{c, M}^{i}$ and $\psi \in D \Sigma_{c_{i}, M_{i}}^{i}$ fixed, we can make

$$
\left\|H\left(\gamma^{\prime}, \psi\right)-H(\gamma, \psi)\right\|_{D \Sigma}=\sum_{l=0}^{i-1} \alpha_{l}\left\|H_{l}\left(\gamma^{\prime}, \psi\right)-H_{l}(\gamma, \psi)\right\|_{0}+\eta\left\|H_{i}\left(\gamma^{\prime}, \psi\right)-H_{i}(\gamma, \psi)\right\|_{1}
$$

as small as we want provided that

$$
\left\|\gamma^{\prime}-\gamma\right\|_{\Sigma}=\max _{1 \leq j \leq i-1}\left(\left\|D^{j} \gamma^{\prime}-D^{j} \gamma\right\|_{0},\left\|\partial_{\theta}^{j} \gamma^{\prime}-\partial_{\theta}^{j} \gamma\right\|_{1}\right)
$$

is small.

Each term of $H_{j}(\gamma, \psi), 0 \leq j \leq i-1$, depends on $\gamma$ through derivatives of $f_{j}$ of order less or equal than $i$, through $\psi_{l}$ in the form $\psi_{l} \circ h$, through $\gamma$ itself, in the form $\partial_{\theta}^{j} \partial_{x}^{l-j} \gamma \circ h(\gamma)$, $l \leq i-1$, and multilinear relations among them. Each term gives a contribution to the difference which can be made small because the involved functions are uniformly continuous on their domains. For instance the term $\partial_{\theta}^{j} \partial_{x}^{l-j} \gamma \circ h(\gamma)$ can be treated as

$$
\begin{aligned}
& \left\|\partial_{\theta}^{j} \partial_{x}^{l-j} \gamma^{\prime} \circ h\left(\gamma^{\prime}\right)-\partial_{\theta}^{j} \partial_{x}^{l-j} \gamma \circ h(\gamma)\right\| \\
& \quad \leq\left\|\partial_{\theta}^{j} \partial_{x}^{l-j} \gamma^{\prime} \circ h\left(\gamma^{\prime}\right)-\partial_{\theta}^{j} \partial_{x}^{l-j} \gamma \circ h\left(\gamma^{\prime}\right)\right\|+\left\|\partial_{\theta}^{j} \partial_{x}^{l-j} \gamma \circ h\left(\gamma^{\prime}\right)-\partial_{\theta}^{j} \partial_{x}^{l-j} \gamma \circ h(\gamma)\right\|
\end{aligned}
$$

which can be made small because $\partial_{\theta}^{j} \partial_{x}^{l-j} \gamma$ is uniformly continuous. The control of $H_{i}\left(\gamma^{\prime}, \psi\right)-$ $H_{i}(\gamma, \psi)$ is more delicate. The terms of $H_{i}$ are of the form:

(a) products of $C^{-1}$ by terms

$$
\begin{gathered}
\partial_{\theta}^{i} g \circ(I, \gamma) \\
\partial_{\theta}^{j} \partial_{v}^{l-j} g(I, \gamma) \partial_{\theta}^{m_{1}} \gamma \cdots \partial_{\theta}^{m_{l-j}} \gamma
\end{gathered}
$$

with $1 \leq l \leq i, 0 \leq j \leq l-1$, and $m_{1}+\ldots+m_{l-j}=i-j$,

$$
\partial_{\theta}^{j} \partial_{x}^{l-j} \gamma \circ h(\gamma)\left[\left(\partial_{\theta}^{m_{1}} h_{1}\right) \cdots\left(\partial_{\theta}^{m_{l-j}} h_{1}\right)\left(\partial_{\theta}^{n_{1}} h_{2}\right) \cdots\left(\partial_{\theta}^{n_{j}} h_{2}\right)\right]
$$


with $1 \leq l \leq i-1,0 \leq j \leq l, m_{i}, n_{i} \geq 1$ and $m_{1}+\cdots+m_{l-j}+n_{1}+\ldots+n_{j}=i$, and

$$
\left(\psi_{j} \circ h\right)\left(\partial_{\theta} h_{1}\right)^{i-j}\left(\partial_{\theta} h_{2}\right)^{j}, \quad 0 \leq j \leq i .
$$

(b) products of $\partial_{\theta}^{l} C^{-1}$ by terms of the form (5.8), and (5.9) with $l \leq i-1$.

We will need the following bounds (if $\gamma \in \Sigma_{\hat{c}, \hat{M}}^{i}$, formally $h_{1}(\gamma)$ is not $C^{i}$ because $\gamma$ is not. However to save computations, we consider the terms $\partial_{\theta}^{i} h_{1}(\gamma)$ below, as their formal expansion from the chain rule but substituting $\partial_{\theta}^{i} \gamma$ by $\psi_{i}$.)

(i) $\left\|\partial_{\theta}^{j} \gamma(z)\right\| \leq\|\gamma\|_{\Sigma}\|x\|, 0 \leq j \leq i-1$,

(ii) $\left\|\partial_{\theta}^{j} \gamma^{\prime}(z)-\partial_{\theta}^{j} \gamma(z)\right\| \leq\left\|\gamma^{\prime}-\gamma\right\|_{\Sigma}\|x\|, \quad 0 \leq j \leq i-1$,

(iii) $\left\|\partial_{\theta}^{j} h_{1}(\gamma)(z)\right\| \leq C\|x\|, \quad 0 \leq j \leq i$,

(iv) for $j=1,2,4,\left\|\partial_{\theta}^{l} f_{j}\left(z, \gamma^{\prime}(z)\right)-\partial_{\theta}^{l} f_{j}(z, \gamma(z))\right\| \leq \chi_{1}\left(\gamma^{\prime}, \gamma\right)\|x\|, 0 \leq l \leq i$. In particular this bound applies to $g$,

(v) $\left\|\partial_{\theta}^{j} h_{1}\left(\gamma^{\prime}\right)(z)-\partial_{\theta}^{j} h_{1}(\gamma)(z)\right\| \leq \chi_{2}\left(\gamma^{\prime}, \gamma\right)\|x\|, \quad 0 \leq j \leq i$,

(vi) $\left\|\partial_{\theta}^{j} \gamma^{\prime}\left(h\left(\gamma^{\prime}\right)\right)(z)-\partial_{\theta}^{j} \gamma(h(\gamma))(z)\right\| \leq \chi_{3}\left(\gamma^{\prime}, \gamma\right)\|x\|, \quad 0 \leq j \leq i-1$.

where $\chi_{1}, \chi_{2}, \chi_{3}$ tend to 0 when $\gamma^{\prime} \rightarrow \gamma$ in $\Sigma^{i}$.

(i), (ii) and (iii) follow easily form the definitions and previous estimates.

(iv) From $f_{j}(w)=\varphi_{j 1}(w) x+\varphi_{j 2}(w) v$ we have

$$
\begin{aligned}
\left\|\partial_{\theta}^{l} f_{j}\left(z, \gamma^{\prime}(z)\right)-\partial_{\theta}^{l} f_{j}(z, \gamma(z))\right\| \leq\left\|\left[\partial_{\theta}^{l} \varphi_{j 1}\left(z, \gamma^{\prime}(z)\right)-\partial_{\theta}^{l} \varphi_{j 1}(z, \gamma(z))\right] x\right\| \\
\quad+\left\|\left[\partial_{\theta}^{l} \varphi_{j 2}\left(z, \gamma^{\prime}(z)\right)-\partial_{\theta}^{l} \varphi_{j 2}(z, \gamma(z))\right] \gamma^{\prime}(z)\right\|+\left\|\partial_{\theta}^{l} \varphi_{j 2}(z, \gamma(z))\left[\gamma^{\prime}(z)-\gamma(z)\right]\right\| \\
\leq \quad\left(\left\|\partial_{\theta}^{l} \varphi_{j 1}\left(z, \gamma^{\prime}(z)\right)-\partial_{\theta}^{l} \varphi_{j 1}(z, \gamma(z))\right\|+\left\|\partial_{\theta}^{l} \varphi_{j 2}\left(z, \gamma^{\prime}(z)\right)-\partial_{\theta}^{l} \varphi_{j 2}(z, \gamma(z))\right\|\left\|\gamma^{\prime}\right\|_{\Sigma}\right. \\
\left.\quad+\left\|\partial_{\theta}^{l} \varphi_{j 2}(z, \gamma(z))\right\|\left\|\gamma^{\prime}-\gamma\right\|_{\Sigma}\right)\|x\| .
\end{aligned}
$$

(v) We decompose $\partial_{\theta}^{j} h_{1}\left(\gamma^{\prime}\right)-\partial_{\theta}^{j} h_{1}(\gamma)$ in telescopic form. We get differences of the form

$$
\left[\partial_{\theta}^{p} \partial_{v}^{l-p} f_{1}\left(z, \gamma^{\prime}(z)\right)-\partial_{\theta}^{p} \partial_{v}^{l-p} f_{1}(z, \gamma(z))\right] \partial_{\theta}^{m_{1}} \gamma \cdots \partial_{\theta}^{m_{l-p}} \gamma
$$

which are bounded by

$$
\left\|\partial_{\theta}^{p} \partial_{v}^{l-p} f_{1}\left(z, \gamma^{\prime}(z)\right)-\partial_{\theta}^{p} \partial_{v}^{l-p} f_{1}(z, \gamma(z))\right\|\|\gamma\|_{\Sigma}^{l-p}\|x\|^{l-p}
$$

(the term with $p=l$ being bounded as in (iv) ) and of the form

$$
\partial_{\theta}^{p} \partial_{v}^{l-p} f_{1}\left(z, \gamma^{\prime}(z)\right)\left[\partial_{\theta}^{m_{1}} \gamma^{\prime} \cdots\left(\partial_{\theta}^{m_{q}} \gamma^{\prime}-\partial_{\theta}^{m_{q}} \gamma\right) \cdots \partial_{\theta}^{m_{l-p}} \gamma\right]
$$

with $p \leq l-1$ which are bounded by $C\|\gamma\|_{\Sigma}^{l-p-1}\|x\|^{l-p-1}\left\|\gamma^{\prime}-\gamma\right\|_{\Sigma}\|x\|$.

In the case $l=i$ also there is the term $\left[\partial_{v}^{i} f_{1}\left(z, \gamma^{\prime}(z)\right)-\partial_{v}^{i} f_{1}(z, \gamma(z))\right] \psi_{i}(z)$ which is bounded by $\left\|\partial_{v}^{i} f_{1}\left(z, \gamma^{\prime}(z)\right)-\partial_{v}^{i} f_{1}(z, \gamma(z))\right\|_{0}\|\psi\|_{D \Sigma}\|x\|$. 
(vi) It follows from $\left\|\partial_{\theta}^{j} \gamma\left(h\left(\gamma^{\prime}\right)\right)-\partial_{\theta}^{j} \gamma\left(h\left(\gamma^{\prime}\right)\right)\right\| \leq\left\|h_{1}\left(\gamma^{\prime}\right)\right\|\left\|\gamma^{\prime}-\gamma\right\|_{\Sigma}$,

$$
\begin{aligned}
& \left\|\partial_{\theta}^{j} \gamma\left(h\left(\gamma^{\prime}\right)\right)-\partial_{\theta}^{j} \gamma(h(\gamma))\right\| \\
& \quad \leq\left\|\partial_{\theta}^{j} \gamma\left(h_{1}\left(\gamma^{\prime}\right), h_{2}\left(\gamma^{\prime}\right)\right)-\partial_{\theta}^{j} \gamma\left(h_{1}\left(\gamma^{\prime}\right), h_{2}(\gamma)\right)\right\|+\left\|\partial_{\theta}^{j} \gamma\left(h_{1}\left(\gamma^{\prime}\right), h_{2}(\gamma)\right)-\partial_{\theta}^{j} \gamma\left(h_{1}(\gamma), h_{2}(\gamma)\right)\right\| \\
& \quad \leq \sup _{\xi}\left\|\partial_{\theta}^{j+1} \gamma\left(h_{1}\left(\gamma^{\prime}\right), \xi\right)\right\|\left\|h_{2}\left(\gamma^{\prime}\right)-h_{2}(\gamma)\right\|+\left\|\partial_{x} \partial_{\theta}^{j} \gamma\right\|\left\|h_{1}\left(\gamma^{\prime}\right)-h_{1}(\gamma)\right\| \\
& \quad \leq\|\gamma\|_{\Sigma}\left\|h_{1}\left(\gamma^{\prime}\right)\right\|\left\|h_{2}\left(\gamma^{\prime}\right)-h_{2}(\gamma)\right\|+\left\|D^{j+1} \gamma\right\|_{0}\left\|h_{1}\left(\gamma^{\prime}\right)-h_{1}(\gamma)\right\|
\end{aligned}
$$

if $j \leq i-2$, and also, since $\gamma(z)=\tilde{\gamma}(z) x$,

$$
\begin{aligned}
\left\|\partial_{\theta}^{i-1} \gamma\left(h\left(\gamma^{\prime}\right)\right)-\partial_{\theta}^{i-1} \gamma(h(\gamma))\right\| \leq & \left\|\partial_{\theta}^{i-1} \tilde{\gamma}\left(h\left(\gamma^{\prime}\right)\right)-\partial_{\theta}^{i-1} \tilde{\gamma}(h(\gamma))\right\|\left\|h_{1}\left(\gamma^{\prime}\right)\right\| \\
& +\left\|\partial_{\theta}^{i-1} \tilde{\gamma}(h(\gamma))\right\|\left\|h_{1}\left(\gamma^{\prime}\right)-h_{1}(\gamma)\right\| .
\end{aligned}
$$

Now we examine the contribution of $(5.7)-(5.10)$ to the difference (5.6). The contribution of (5.7) is evaluated as in (iv).

The term (5.8) must be decomposed in telescopic form and hence we have to consider differences

$$
\|x\|^{-1}\left[\partial_{\theta}^{j} \partial_{v}^{l-j} g\left(I, \gamma^{\prime}\right)-\partial_{\theta}^{j} \partial_{v}^{l-j} g(I, \gamma)\right] \partial_{\theta}^{m_{1}} \gamma \cdots \partial_{\theta}^{m_{l-j}} \gamma, \quad 1 \leq l \leq i, \quad 0 \leq j \leq l-1
$$

and

$$
\|x\|^{-1} \partial_{\theta}^{j} \partial_{v}^{l-j} g(I, \gamma) \partial_{\theta}^{m_{1}} \gamma^{\prime} \cdots\left[\partial_{\theta}^{m_{q}} \gamma^{\prime}-\partial_{\theta}^{m_{q}} \gamma\right] \cdots \partial_{\theta}^{m_{l-j}} \gamma
$$

For (5.13) note that $l-j \geq 1$ and then by (i) the term is bounded and by (iv) it can be made small. The contribution of the terms (5.9) decompose as sums of the form

$$
\left[\partial_{\theta}^{j} \partial_{x}^{l-j} \gamma^{\prime}\left(h\left(\gamma^{\prime}\right)\right)-\partial_{\theta}^{j} \partial_{x}^{l-j} \gamma(h(\gamma))\right]\left[\left(\partial_{\theta}^{m_{1}} h_{1}\right) \cdots\left(\partial_{\theta}^{m_{l-j}} h_{1}\right)\left(\partial_{\theta}^{n_{1}} h_{2}\right) \cdots\left(\partial_{\theta}^{n_{j}} h_{2}\right)\right]
$$

and terms of the form (5.9) where one substitutes $\partial_{\theta}^{m_{q}} h_{1}(\gamma)$ by $\partial_{\theta}^{m_{q}} h_{1}\left(\gamma^{\prime}\right)-\partial_{\theta}^{m_{q}} h_{1}(\gamma)$ or $\partial_{\theta}^{n_{q}} h_{2}(\gamma)$ by $\partial_{\theta}^{n_{q}} h_{2}\left(\gamma^{\prime}\right)-\partial_{\theta}^{n_{q}} h_{2}(\gamma)$. The left factor of $(5.15)$ is bounded by $\left\|D^{l} \gamma^{\prime}-D^{l} \gamma\right\|+$ $\left\|D^{l+1} \gamma\right\|\left\|h\left(\gamma^{\prime}\right)-h(\gamma)\right\|$. If $l-j \geq 1$ this bound is enough because the right factor contains at least one derivative of $\partial_{\theta}^{i} h_{1}$ whose bound has a factor $\|x\|$. If $l-j=0$ one must use (vi). The other differences, if $j<l$, contain a derivative $\partial_{\theta}^{i} h_{1}$ and/or a difference $\partial_{\theta}^{m q} h_{1}\left(\gamma^{\prime}\right)-\partial_{\theta}^{m q} h_{1}(\gamma)$ which tends to zero. If $j=l$ we have the factor $\partial_{\theta}^{l} \gamma(h(\gamma))$ which is bounded by $\|\gamma\| \Sigma\left\|h_{1}(\gamma)\right\| \leq$ $\|\gamma\|_{\Sigma} N_{2}\|x\|$. Finally the contribution of (5.10) can be treated analogously to the one of (5.9).

Lemma 5.6 There exist $\delta_{0}, \mu_{0}>0$ such that for $0<\delta<\delta_{0}$ and $\nu \in \mathcal{B}_{\mu_{0}}^{s}$, the map $H(\gamma,$.$) is$ a contraction on $D \sum_{c_{i}, M_{i}}^{i}$, uniform with respect to $\gamma \in \sum_{\hat{c}, \hat{M}^{*}}^{i}$.

Proof of Lemma 5.6. In order to bound $\left\|H(\gamma, \psi)-H\left(\gamma, \psi^{\prime}\right)\right\|_{D \Sigma}$ we first consider the difference between their components, $H_{j}(\gamma, \psi)$ and $H_{j}\left(\gamma, \psi^{\prime}\right)$ :

$$
\begin{aligned}
& \left\|H_{j}(\gamma, \psi)-H_{j}\left(\gamma, \psi^{\prime}\right)\right\| \leq \beta\left(\left(L+c_{1} L+M_{1}\left(\lambda^{-1}+L\right)\left(K_{\omega}+K_{f}\right) \delta\right)\left\|\psi_{j}-\psi_{j}^{\prime}{ }_{j}\right\|\right. \\
& \quad+\left(N_{5} / \beta\right)\|x\|\left\|\psi_{0} \circ h-\psi_{0}^{\prime} \circ h\right\|+\ldots+\left(N_{3}^{j} N_{1}^{i-j}+\left(N_{5} / \beta\right)\|x\|\right)\left\|\psi_{j} \circ h-\psi_{j}^{\prime} \circ h\right\|+\ldots \\
& \left.\quad+\left(N_{5} / \beta\right)\left\|\psi_{i} \circ h-\psi_{i}^{\prime} \circ h\right\|\right) .
\end{aligned}
$$


Therefore, we have that

$$
\begin{aligned}
& \sum_{l=0}^{i-1} \alpha_{l}\left\|H_{l}(\gamma, \psi)(z)-H_{l}\left(\gamma, \psi^{\prime}\right)(z)\right\|+\eta\|x\|^{-1}\left\|H_{i}(\gamma, \psi)(z)-H_{i}\left(\gamma, \psi^{\prime}\right)(z)\right\| \\
& \leq \quad N_{5}\|x\|\left[\left\|\psi_{0}-\psi_{0}^{\prime}\right\|\left(\alpha_{0}+\ldots+\alpha_{i-1}\right)+\left\|\psi_{1}-\psi_{1}^{\prime}\right\|\left(\alpha_{1}+\ldots+\alpha_{i-1}\right)+\ldots\right. \\
& \left.\quad+\left\|\psi_{i-1}-\psi_{i-1}^{\prime}\right\| \alpha_{i-1}\right]+\Lambda \sum_{l=0}^{i-1} \alpha_{l}\left\|\psi_{l}-\psi_{l}^{\prime}\right\|+N_{5}\left[\alpha_{0}\left\|\psi_{1}-\psi_{1}^{\prime}\right\|\right. \\
& \left.\quad+\left(\alpha_{0}+\alpha_{1}\right)\left\|\psi_{2}-\psi_{2}^{\prime}\right\|+\ldots+\left(\alpha_{0}+\ldots+\alpha_{i-1}\right)\left\|\psi_{i}-\psi_{i}^{\prime}\right\|\right] \\
& \quad+\eta N_{5}\left[\left\|\psi_{0}-\psi_{0}^{\prime}\right\|+\ldots+\left\|\psi_{i}-\psi_{i}^{\prime}\right\|\right]+\Lambda \eta\left\|\psi_{i}-\psi_{i}^{\prime}\right\|_{1}
\end{aligned}
$$

where we have used that

$$
\beta N_{3}^{l} N_{1}^{i-l}+\beta\left(L+c_{1} L+M_{1}\left(\lambda^{-1}+L\right)\left(K_{\omega}+K_{f}\right) \delta\right)<\Lambda, \quad 0 \leq l \leq i-1,
$$

if $\delta, \mu$ are small. Using that $\left(\alpha_{0}+\ldots+\alpha_{l-1}\right)=\eta \alpha_{l}$, and

$$
\left\|\psi_{i} \circ h(z)-\psi_{i}^{\prime} \circ h(z)\right\| \leq\left\|h_{1}(z)\right\|\left\|\psi_{i}-\psi_{i}^{\prime}\right\|_{1} \leq\left(\lambda^{-1}+L\right)\|x\|\left\|\psi_{i}-\psi_{i}^{\prime}\right\|_{1}
$$

and that if $\delta$ is small $\left(\alpha_{l}+\ldots+\alpha_{i-1}\right) \delta^{1 / 2}<\alpha_{l}, 0 \leq l \leq i-2$, we get that

$$
\left\|H(\gamma, \psi)-H\left(\gamma, \psi^{\prime}\right)\right\|_{D \Sigma} \leq \sum_{l=0}^{i-1} \alpha_{l}\left\|\psi_{l}-\psi_{l}^{\prime}\right\|_{0}\left(N_{5} \delta^{1 / 2}+\Lambda+N_{5} \eta+\eta N_{5}\right)+\Lambda \eta\left\|\psi_{i}-\psi_{i}^{\prime}\right\|_{1} .
$$

Then if $\delta$ is small enough $H(\gamma,$.$) is a contraction.$

End of the proof of the theorem Once we have proved the induction hypotheses we easily get inductively that $\gamma^{s}$ is $C^{k-1}$. A final application of the Fiber Contraction Theorem to $\Gamma=(G, H): \sum_{\hat{c}, \hat{M}}^{k} \times D \Sigma_{c_{k}, M_{k}}^{k} \longrightarrow \sum_{\hat{c}, \hat{M}}^{k} \times D \sum_{c_{k}, M_{k}}^{k}$ proves that $\gamma^{s}$ is of class $C^{k}$.

It remains to be proved the $C^{k}$ dependence on $\nu$. This is accomplished by considering the map $\tilde{F}$ defined by $\tilde{F}(w, \nu)=(F(w, \nu), \nu)$ as we did in the Lipschitz case.

\section{The $C^{\infty}$ case}

Here we consider $F_{\nu}: \mathcal{M}_{\delta} \longrightarrow \mathcal{M}$ defined by (2.1) in the $C^{\infty}$ setting. We ask for the analogous $C^{\infty}$ conditions but now we have to restrict the dynamics on the torus to a rotation. Precisely, let $\lambda, \beta, K_{l}$ and $K_{\omega}$ be positive constants such that

$$
\lambda>1, \quad \beta \geq 1, \quad \lambda^{-1} \beta<1,
$$

and let $L(\delta, \mu)$ and $M(\delta)$ be continuous non-negative functions with $L(0,0)=0, M(0)=0$, verifying

HI1 The functions $A_{ \pm}, B, \omega$ and $f$ are $C^{\infty}$ both with respect to $w$ and $\nu$.

$\mathrm{HI} 2=\mathrm{HC} 2$

HI3 For $j=1,2,4$ the functions $f_{j}$ verify that $f_{j}(0,0, \theta, 0, \nu)=0$ and

$$
\left\|\partial_{\theta} f_{j}(w, \nu)\right\| \leq K_{m}\|(x, y, r)\|, \quad\left\|D f_{j}(w, \nu)\right\| \leq L(\delta, \mu), \quad(w, \nu) \in \mathcal{M}_{\delta} \times \mathcal{B}_{\mu}^{s} .
$$


HI4 $f_{3}(0,0, \theta, 0, \nu)=0$ and $\left\|\partial_{\theta} f_{3}(w, \nu)\right\| \leq M(\delta)$.

$\mathbb{T}_{0}=\{x=0, y=0, r=0\}$ is invariant by $F_{\nu}$ and the map $\Phi_{\nu}=F_{3 \nu} \mid \mathbb{T}_{0}$ is a rotation with frequency vector $\omega(0,0,0)$. In particular $\partial_{\theta} \Phi_{\nu}(\theta)=I d$.

Remark 6.1 In the $C^{k}$ case, the condition $\lambda^{-1} \beta\left(1+K_{\theta}\right)^{k}<1$ is in fact a bound for $k$. Here we substitute the bound $K_{\theta}$ by $M(\delta)$. In this way, for any $k$, we can choose $\delta$ such that the previous condition is satisfied.

The main result of this section is

Theorem 6.2 Let $F_{\nu}: \mathcal{M}_{\delta} \rightarrow \mathcal{M}$ satisfy hypotheses HIt to HI4 and $F_{\nu}$ invertible on $W^{s}$ (for that it is sufficient that $A_{-}(\theta)$ is invertible.) Then there exist $\delta_{0}$ and $\mu_{0}$ such that, if $0<\delta<\delta_{0}$ and $\|\nu\|<\mu_{0}$, the function $\gamma_{\nu}^{s}(x, \theta)$ given by Theorem 2.1 whose graph is the stable invariant manifold of $\mathbb{T}_{0}$ is $C^{\infty}$. Moreover if we ask

HI5 $\left\|\partial_{\nu} f_{j}(w, \nu)\right\| \leq K_{m}\|(x, y, r)\|$, for $j=1,2,4$ and $\left\|\partial_{\nu} f_{3}(w, \nu)\right\| \leq M(\delta)$

then $\gamma^{s}$ is $C^{\infty}$ with respect to $(x, \theta, \nu)$.

Proof of Theorem 6.2. It is clear that hypotheses HI1-HI4 imply HC1-HC4 for $\|x\|<\delta_{k}$ and $\|\nu\|<\mu_{k}$. Then, for each $k$ it is possible to find $\delta_{k}$ and $\mu_{k}$ such that the function $\gamma_{\nu}^{s}(x, \theta)$ is $C^{k}$. However, the numbers $\delta_{k}$ and $\mu_{k}$ may decrease to 0 . This is not the case of $\delta_{k}$, since, by Remark $3.5, \delta_{k}$ can be restored to its original size, $\delta_{0}$. In order to see that there exists a value $\mu_{0}$ valid for all $k$ we make a change of variables to put in evidence that the dependence of $\nu$ is such that it is not necessary to restrict $\mu$ as $k$ increases.

Lemma 6.3 Let $F_{\nu}$ be the map defined by (2.1), satisfying hypotheses HI1 - HI4. Then there exist $\mu_{0}>0$ and a $C^{\infty}$ change of variables, $C_{\nu},\|\nu\|<\mu_{0}$, (which also depends $C^{\infty}$ in $\nu$ ) such that the map $\tilde{F}_{\nu}=C_{\nu}^{-1} \circ F_{\nu} \circ C_{\nu}$ can be written in the form (2.1), satisfying HI1 $H I_{4}$, possibly with slightly different constants $\lambda, \beta$, with $L(\delta, \mu)=L^{*}(\delta)$ independent on $\nu$.

Assume for the moment that Lemma 6.3 is proved. After performing the change of variables, the map verifies hypotheses HI1-HI4 with bounds uniformly valid on $\mu$. This implies that $\mu_{k}$ can be taken independent on $k$.

\subsection{Proof of Lemma 6.3}

Lemma 6.3 is a consequence of the following Lemma

Lemma 6.4 Let $F_{\nu}$ be the map defined by (2.1), satisfying hypotheses HII-HI4. Then there exist $\mu_{0}>0$ and $a C^{\infty}$ change of variables, $C_{\nu},\|\nu\|<\mu_{0}$, such that the map $\tilde{F}_{\nu}=C_{\nu}^{-1} \circ F_{\nu} \circ C_{\nu}$ has the form

$$
\tilde{F}_{\nu}(x, y, \theta, r)=\left(\tilde{A}_{-}(\theta, \nu) x, \tilde{A}_{+}(\theta, \nu) y, \theta+\tilde{\omega}(x, y, r), \tilde{B}(\theta, \nu) r\right)+\tilde{f}(x, y, \theta, r, \nu),
$$

satisfies $\mathrm{HI}_{1}-\mathrm{HI}_{4}$ and its derivative on the torus has the following structure

$$
D \tilde{F}_{\nu}(0,0, \theta, 0)=\left(\begin{array}{cccc}
R_{11} & 0 & 0 & 0 \\
0 & R_{22} & 0 & 0 \\
R_{31} & R_{32} & R_{33} & R_{34} \\
0 & 0 & 0 & R_{44}
\end{array}\right)
$$


with $R_{11}=\tilde{A}_{-}(\theta, \nu), R_{22}=\tilde{A}_{+}(\theta, \nu), R_{33}=I d, R_{44}=\tilde{B}(\theta, \nu)$ and $\tilde{A}_{ \pm}(\theta, 0)=A_{ \pm}(\theta)$, $\tilde{B}(\theta, 0)=B(\theta)$. If $F_{\nu}$ satisfies HI1-HI5, $C_{\nu}$ is $C^{\infty}$ with respect to $(w, \nu)$.

Proof of Lemma 6.4. We seek a change $C$ of the form

$$
C(x, y, \theta, r)=\left(x+a_{12} y+a_{14} r, y+a_{21} x+a_{24} r, \theta, r+a_{41} x+a_{42} y\right)
$$

where $a_{i j}=a_{i j}(\theta, \nu)$ are linear functions, for instance $a_{12}(\theta, \nu) \in L\left(\mathbb{R}^{p}, \mathbb{R}^{m}\right)$.

We impose the condition

$$
D F(0,0, \theta, 0) D C(0,0, \theta, 0)=D C(F(0,0, \theta, 0)) D \tilde{F}(0,0, \theta, 0)
$$

where $D \tilde{F}(0,0, \theta, 0)$ has the required structure (6.1). This gives the following equations

$$
\begin{aligned}
& \left(A_{-}+\partial_{x} f_{1}\right) a_{12}+\partial_{y} f_{1}+\partial_{r} f_{1} a_{42}=a_{12}^{*} R_{22}=a_{12}^{*}\left[\partial_{x} f_{2} a_{12}+A_{+}+\partial_{y} f_{2}+\partial_{r} f_{2} a_{42}\right] \\
& \left(A_{-}+\partial_{x} f_{1}\right) a_{14}+\partial_{y} f_{1} a_{24}+\partial_{r} f_{1}=a_{14}^{*} R_{44}=a_{14}^{*}\left[\partial_{x} f_{4} a_{14}+\partial_{y} f_{4} a_{24}+B+\partial_{r} f_{4}\right] \\
& \partial_{x} f_{2}+\left(A_{+}+\partial_{y} f_{2}\right) a_{21}+\partial_{r} f_{2} a_{41}=a_{21}^{*} R_{11}=a_{21}^{*}\left[A_{-}+\partial_{x} f_{1}+\partial_{y} f_{1} a_{21}+\partial_{r} f_{1} a_{41}\right] \\
& \partial_{x} f_{2} a_{14}+\left(A_{+}+\partial_{y} f_{2}\right) a_{24}+\partial_{r} f_{2}=a_{24}^{*} R_{44}=a_{24}^{*}\left[\partial_{x} f_{4} a_{14}+\partial_{y} f_{4} a_{24}+B+\partial_{r} f_{4}\right] \\
& \partial_{x} f_{4}+\partial_{y} f_{4} a_{21}+\left(B+\partial_{r} f_{4}\right) a_{41}=a_{41}^{*} R_{11}=a_{41}^{*}\left[A_{-}+\partial_{x} f_{1}+\partial_{y} f_{1} a_{21}+\partial_{r} f_{1} a_{41}\right] \\
& \partial_{x} f_{4} a_{12}+\partial_{y} f_{4}+\left(B+\partial_{r} f_{4}\right) a_{42}=a_{42}^{*} R_{22}=a_{42}^{*}\left[\partial_{x} f_{2} a_{12}+A_{+}+\partial_{y} f_{2}+\partial_{r} f_{2} a_{42}\right]
\end{aligned}
$$

where the derivatives are evaluated at $(0,0, \theta, 0, \nu)$ and $a_{i j}^{*}$ means $a_{i j}\left(\Phi_{\nu}(\theta)\right)$. The functions $a_{i j}$ appear in pairs. For instance to get $a_{12}$ and $a_{42}$ we have to consider the first and the sixth equations. We concentrate our attention to those equations, the other cases being analogous. To simplify the notation we write $a$ for $a_{12}$ and $b$ for $a_{42}$. They can written as

$$
\begin{aligned}
\mathcal{L}_{1} a & =\mathcal{R}_{1}(a, b), \\
\mathcal{L}_{2} b & =\mathcal{R}_{2}(a, b)
\end{aligned}
$$

where $\mathcal{L}_{1}$ and $\mathcal{L}_{2}$ are given by the linear maps: $\mathcal{L}_{1}: C^{\infty}\left(\mathbb{T}^{n}, L\left(\mathbb{R}^{p}, \mathbb{R}^{m}\right)\right) \longrightarrow C^{\infty}\left(\mathbb{T}^{n}, L\left(\mathbb{R}^{p}, \mathbb{R}^{m}\right)\right)$ with

$$
\mathcal{L}_{1}(a)=A_{-} a-a^{*} A_{+}
$$

and $\mathcal{L}_{2}: C^{\infty}\left(\mathbb{T}^{n}, L\left(\mathbb{R}^{p}, \mathbb{R}^{q}\right)\right) \longrightarrow C^{\infty}\left(\mathbb{T}^{n}, L\left(\mathbb{R}^{p}, \mathbb{R}^{q}\right)\right)$ with

$$
\mathcal{L}_{2}(b)=B b-b^{*} A_{+}
$$

and

$$
\mathcal{R}_{1}(a, b)=a^{*}\left(\partial_{x} f_{2} a+\partial_{y} f_{2}+\partial_{r} f_{2} b\right)-\partial_{x} f_{1} a-\partial_{y} f_{1}-\partial_{r} f_{1} b
$$

and

$$
\mathcal{R}_{2}(a, b)=b^{*}\left(\partial_{x} f_{2} a+\partial_{y} f_{2}+\partial_{r} f_{2} b\right)-\partial_{x} f_{4} a-\partial_{y} f_{4}-\partial_{r} f_{4} b .
$$

Now we prove that $\mathcal{L}_{1,2}$ are invertible. The condition $\mathcal{L}_{1}(a)=c$ is equivalent to the fixed point equation

$$
a=\left[A_{-} a A_{+}^{-1}-c A_{+}^{-1}\right] \circ \Phi^{-1} .
$$

We define the right hand side term of (6.4) by $\Gamma(a)$. Clearly if $a \in C^{0}, \Gamma(a) \in C^{0}$. First we prove that $\Gamma$ is a contraction on $C^{0}$. Indeed

$$
\left\|\Gamma\left(a^{\prime}\right)-\Gamma(a)\right\| \leq\left\|A_{-}\right\|\left\|a^{\prime}-a\right\|\left\|A_{+}^{-1}\right\| \leq \lambda^{-2}\left\|a^{\prime}-a\right\| .
$$


Let $a_{\infty} \in C^{0}$ the unique fixed point of $\Gamma$. To prove that $a_{\infty}$ is $C^{\infty}$ we prove inductively that it is $C^{j}, j \geq 1$, by using the Fiber Contraction Theorem. For $j=1$ we define $\Delta \Gamma$ from $C^{0}\left(\mathbb{T}^{n}, L\left(\mathbb{R}^{p}, \mathbb{R}^{m}\right)\right) \times C^{0}\left(\mathbb{T}^{n}, L\left(\mathbb{R}^{n}, L\left(\mathbb{R}^{p}, \mathbb{R}^{m}\right)\right)\right)$ to itself as the operator obtained derivating formally $\Gamma$ and putting $\Delta a$ instead of $\partial_{\theta} a$. We have

$$
\Delta \Gamma(a, \Delta a)=\left(\left(\partial_{\theta} A_{-}\right) a A_{+}^{-1}+A_{-} \Delta a A_{+}^{-1}+A_{-} a \partial_{\theta} A_{+}^{-1}-\partial_{\theta} c A_{+}^{-1}-c \partial_{\theta} A_{+}^{-1}\right) \circ \Phi^{-1} .
$$

In this way

$$
\partial_{\theta}(\Gamma(a))=\Delta \Gamma\left(a, \partial_{\theta} a\right) .
$$

One readily checks that $\Delta \Gamma(a,$.$) is a contraction and that \Delta \Gamma(., \Delta a)$ is continuous.

Using the arguments of Section 4 we get that $a_{\infty}$ is $C^{1}$ and furthermore that it is an attracting fixed point of $\Gamma$ in $C^{1}$.

Assuming by induction that $\Gamma$ has an attracting fixed point in $C^{j-1}$ we define now

$$
\Delta \Gamma: C^{j-1}\left(\mathbb{T}^{n}, L\left(\mathbb{R}^{p}, \mathbb{R}^{m}\right)\right) \times C^{0}\left(\mathbb{T}^{n}, L^{j}\left(\mathbb{R}^{n}, L\left(\mathbb{R}^{p}, \mathbb{R}^{m}\right)\right)\right) \longrightarrow C^{0}\left(\mathbb{T}^{n}, L^{j}\left(\mathbb{R}^{n}, L\left(\mathbb{R}^{p}, \mathbb{R}^{m}\right)\right)\right)
$$

by derivating formally $j$ times $\Gamma(a)$ with respect to $\theta$ and putting $\Delta a$ instead of $\partial_{\theta}^{j} a$. Now $\partial_{\theta}^{j} a_{i}=\partial_{\theta}^{j}\left(\Gamma\left(a_{i-1}\right)\right)=\Delta \Gamma\left(a_{i-1}, \partial_{\theta}^{j} a_{i-1}\right)$. Clearly $\Delta \Gamma$ is the sum of two terms $T_{1}, T_{2}$ where $T_{1}(a, \Delta a)=\left(A_{-} \Delta a A_{+}^{-1}\right) \circ \Phi^{-1}$ and $T_{2}$ depends on the derivatives of $a$ up to order $j-1$ and involves the derivatives with respect to $\theta$ of $A_{-}, A_{+}, c$ up to order $j$. As before $\Delta \Gamma(a,$.$) is a$ contraction whose contraction factor does not depend on $j$ (since $\partial_{\theta} \Phi=I d$ ) and $\Delta \Gamma(., \Delta a$ ) is continuous. Then $(\Gamma, \Delta \Gamma)$ from $C^{j-1} \times C^{0}$ to itself has a unique attracting fixed point. Taking $a_{0} \in C^{j}$, and using again the results fo Section 4 we get that $\Gamma^{i}\left(a_{0}\right)$ converges to a $C^{j}$ function which means that $a_{\infty}$ is $C^{j}$.

We also shall need the inverse of the operator $\left(\Delta^{j} \mathcal{L}\right)$ from $C^{\infty}\left(\mathbb{T}^{n}, L^{j}\left(\mathbb{R}^{n}, L\left(\mathbb{R}^{p}, \mathbb{R}^{m}\right)\right)\right)$ to itself defined by

$$
\left(\Delta^{j} \mathcal{L}\right)(\Delta a)=A_{-} \Delta a-\Delta a^{*} A_{+}
$$

where $\Delta a^{*}=\Delta a \circ \Phi$. Its invertibility is obtained in the same way and using that $\left\|A_{-}\right\|\left\|A_{+}^{-1}\right\|<$ 1.

We are interested in calculating $\partial_{\theta}^{j}\left(\mathcal{L}_{1}^{-1} c\right), j \geq 1$. Since we already know that $\mathcal{L}_{1}^{-1} c$ is $C^{\infty}$ we can derivate implicitly $\mathcal{L}_{1} a=c$. We have

$$
\partial_{\theta}^{j} c=A_{-} \partial_{\theta}^{j} a+\sum_{i=1}^{j}\left(\begin{array}{l}
j \\
i
\end{array}\right)\left(\partial_{\theta}^{i} A_{-}\right)\left(\partial_{\theta}^{j-i} a\right)-\left(\partial_{\theta}^{j} a\right) \circ \Phi A_{+}-\sum_{i=1}^{j}\left(\begin{array}{l}
j \\
i
\end{array}\right)\left(\partial_{\theta}^{j-i} a^{*}\right)\left(\partial_{\theta}^{j} A_{+}\right) .
$$

Then

$$
\partial_{\theta}^{j}\left(\mathcal{L}_{1}^{-1} c\right)=\left(\Delta^{j} \mathcal{L}\right)^{-1}\left[\partial_{\theta}^{j} c-\sum_{i=1}^{j}\left(\partial_{\theta}^{i} A_{-}\right)\left(\partial_{\theta}^{j-i} a\right)-\sum_{i=1}^{j}\left(\partial_{\theta}^{j-i} a^{*}\right)\left(\partial_{\theta}^{j} A_{+}\right)\right]
$$

where $\left(\Delta^{j} \mathcal{L}\right)(\Delta a)=A_{-} \Delta a-\Delta a \circ \Phi\left(\partial_{\theta} \Phi\right)^{j} A_{+}$which is invertible and $\left\|\left(\Delta^{j} \mathcal{L}\right)^{-1}\right\| \leq(\lambda-$ $\left.\lambda^{-1}\right)^{-1}$, which is independent of $j$.

To invert $\mathcal{L}_{2}$ we consider the equation $\mathcal{L}_{2}(b)=c$ in its equivalent form

$$
b=\left(B b A_{+}^{-1}-c A_{+}^{-1}\right) \circ \Phi^{-1} .
$$

Since $\|B\|\left\|A_{+}^{-1}\right\|<1$ we can proceed as before to obtain $b$ as a $C^{\infty}$ function depending linearly in $c$. 
We rewrite $(6.2)$ in the form

$$
\begin{aligned}
a & =\mathcal{L}_{1}^{-1} \mathcal{R}_{1}(a, b) \\
b & =\mathcal{L}_{2}^{-1} \mathcal{R}_{2}(a, b)
\end{aligned}
$$

Now we define $\Gamma=\left(\Gamma_{1}, \Gamma_{2}\right)$ from $C^{j}\left(\mathbb{T}^{n}, L\left(\mathbb{R}^{p}, \mathbb{R}^{m}\right)\right) \times C^{j}\left(\mathbb{T}^{n}, L\left(\mathbb{R}^{p}, \mathbb{R}^{q}\right)\right)$ to itself by letting $\Gamma_{1}(a, b)$ and $\Gamma_{2}(a, b)$ be the right hand side terms of $(6.6)$ and $(6.7)$ respectively. We take the maximum norm in the product space. When $j=0, \Gamma$ sends the unit ball to the unit ball. Indeed since all terms of $\Gamma_{1}$ and $\Gamma_{2}$ contain derivatives of $f_{j}$ we have that if $\mu$ is small enough the image has norm less that one. Moreover if $\|(a, b)\|_{C^{0}}<1, \Gamma$ is a contraction. For instance we compute:

$$
\begin{aligned}
& \left\|\Gamma_{1}\left(a^{\prime}, b^{\prime}\right)-\Gamma_{1}(a, b)\right\| \\
& \leq\left\|\mathcal{L}_{1}^{-1}\right\|\left(\left\|a^{*}-a^{*}\right\|\left\|\left(\partial_{x} f_{2}+\partial_{r} f_{2}\right) a^{\prime}+\partial_{y} f_{2}\right\|+\left\|a^{*}\right\|\left\|\partial_{x} f_{2}+\partial_{r} f_{2}\right\|\left\|a^{\prime}-a\right\|\right. \\
& \left.\quad+\left\|\partial_{x} f_{1}\right\|\left\|a^{\prime}-a\right\|+\left\|\partial_{r} f_{1}\right\|\left\|b^{\prime}-b\right\|\right) .
\end{aligned}
$$

In fact there exist $M>0$ such that if $\|(a, b)\| \leq M \mu$ then $\|\Gamma(a, b)\| \leq M \mu$. This means that the fixed point of $\Gamma$ actually belongs to the ball of radius $M \mu$.

Derivating formally $\Gamma(a, b) j$-times with respect to $\theta$ and substituting $\partial_{\theta}^{j} a$ and $\partial_{\theta}^{j} b$ by $\Delta a$ and $\Delta b$ respectively we get the map $\Delta \Gamma(a, b, \Delta a, \Delta b)$ and we consider

$$
(\Gamma, \Delta \Gamma): E_{1} \times E_{2} \longrightarrow E_{1} \times E_{2}
$$

where

$$
E_{1}=C^{j-1}\left(\mathbb{T}^{n}, L\left(\mathbb{R}^{p}, \mathbb{R}^{m}\right)\right) \times C^{j-1}\left(\mathbb{T}^{n}, L\left(\mathbb{R}^{p}, \mathbb{R}^{q}\right)\right)
$$

and

$$
E_{2}=C^{0}\left(\mathbb{T}^{n}, L^{j}\left(\mathbb{R}^{n}, L\left(\mathbb{R}^{p}, \mathbb{R}^{m}\right)\right)\right) \times C^{0}\left(\mathbb{T}^{n}, L^{j}\left(\mathbb{R}^{n}, L\left(\mathbb{R}^{p}, \mathbb{R}^{q}\right)\right)\right)
$$

and $\Delta \Gamma=T_{1}+T_{2}$ with $T_{1}=\left(T_{1}^{1}, T_{1}^{2}\right)$

$$
\begin{aligned}
T_{1}^{1}(a, b, \Delta a, \Delta b)=\left(\Delta^{j} \mathcal{L}_{1}\right)^{-1}\left[(\Delta a)^{*}\left(\partial_{\theta} \Phi\right)^{j}\left(\partial_{x} f_{2} a+\partial_{y} f_{2}+\partial_{r} f_{2} b\right)\right. & \\
& \left.+a^{*}\left(\partial_{x} f_{2} \Delta a+\partial_{r} f_{2} \Delta b\right)-\partial_{x} f_{1} \Delta a+\partial_{r} f_{1} \Delta b\right] \\
T_{1}^{2}(a, b, \Delta a, \Delta b)=\left(\Delta^{j} \mathcal{L}_{2}\right)^{-1}\left[(\Delta b)^{*}\left(\partial_{\theta} \Phi\right)^{j}\left(\partial_{x} f_{2} a+\partial_{y} f_{2}+\partial_{r} f_{2} b\right)\right. & \\
& \left.+b^{*}\left(\partial_{x} f_{2} \Delta a+\partial_{r} f_{2} \Delta b\right)-\partial_{x} f_{4} \Delta a+\partial_{r} f_{4} \Delta b\right]
\end{aligned}
$$

and $T_{2}$ only depends on $a, b$ and their derivatives up to order $j-1$. By the induction hypothesis $\Gamma$ has an attracting fixed point in $C^{j-1}\left(\mathbb{T}, L\left(\mathbb{R}^{p}, \mathbb{R}^{m}\right)\right), \Delta \Gamma(., ., \Delta a, \Delta b)$ is continuous and $\Delta \Gamma(a, b, . .$.$) is a contraction. Then, as before, (\Gamma, \Delta \Gamma)$ has a unique attracting fixed point and then $(a, b) \in C^{j}$.

If we add the hypothesis HI5 we can apply the above results to $(w, \nu) \mapsto\left(F_{\nu}(w), \nu\right)$, considering $\nu$ as a new angle as discussed in the previous sections.

\section{Lambda Lemma}

\subsection{The Settings and the Lambda Lemma}

In this section we consider a map $F_{\nu}$ of the form (2.1) verifying hypotheses $\mathrm{HC} 1$ to $\mathrm{HC} 3$ with $k \geq 2$ and 
HC4' The function $f_{3}$ has the form

$$
f_{3}(w, \nu)=\varphi_{31}(w, \nu) x+\varphi_{32}(w, \nu) v, \quad v=(y, r),
$$

with $\varphi_{3 i}$ of class $C^{k}$ with respect to $\theta$, and

$$
\left\|\partial_{\theta} f_{3}(w, \nu)\right\| \leq K_{\theta}\|(x, y, r)\|, \quad\left\|D f_{j}(w, \nu)\right\| \leq L_{f}\|(x, y, r)\|, \quad(w, \nu) \in \mathcal{M}_{\delta} \times \mathcal{B}_{\mu}^{s} .
$$

In particular, $f_{3}(0,0, \theta, 0)=0$, which implies that $F_{\nu} \mid \mathbb{T}_{0}$ is simply the rotation $\theta \mapsto$ $\theta+\omega(0,0,0)$. We will assume that $\omega_{0}=\omega(0,0,0)$ is non-resonant, i.e. $\forall k \in\left(\mathbb{Z}_{+}\right)^{n}, \omega_{0} \cdot k \neq 0$.

Moreover, for each $\theta \in \mathbb{T}^{n}$, we suppose that the linear maps $A_{-}(\theta), A_{+}(\theta)$ and $B(\theta)$ are invertible and that $\|B(\theta)\|<\beta$. Under these hypotheses, it is not difficult to see that the map $F_{\nu}$ is invertible in a neighborhood of $\mathbb{T}_{0}$ and its inverse can be written in the same form (2.1), verifying $\mathrm{HC} 1$ to $\mathrm{HC} 3$ and $\mathrm{HC} 4$ '. To show this requires to redo the proof of the Inverse Function Theorem under these hypotheses. It is convenient to work in the space $\Sigma^{i}, D \Sigma^{i}$, $i \leq 2$, of Section 3. In this way, Theorem 3.1 can be applied to both $F_{\nu}$ and $F_{\nu}^{-1}$, which leads to the existence of invariant stable and unstable manifolds, $W^{s, u}$, of dimensions $m+n$ and $p+n$, respectively. These invariant manifolds are given by $W^{s}=\left\{(y, r)=\gamma^{s}(x, \theta)\right\}$ and $W^{u}=\left\{(x, r)=\gamma^{u}(y, \theta)\right\}$, where the functions $\gamma^{u, s}$ also verify

$$
\gamma^{s}(x, \theta)=\tilde{\gamma}^{s}(x, \theta) x, \quad \partial_{\theta} \gamma^{s}(x, \theta)=\tilde{\psi}_{1}(x, \theta) x, \quad \partial_{\theta}^{2} \gamma^{s}(x, \theta)=\tilde{\psi}_{2}(x, \theta) x,
$$

with $\tilde{\gamma}^{s}, \tilde{\psi}_{1}$ and $\tilde{\psi}_{2}$ continuous, and analogously for $\gamma^{u}$. It is not obvious that $\tilde{\psi}_{1}=\partial_{\theta} \tilde{\gamma}^{s}$ and $\tilde{\psi}_{2}=\partial_{\theta}^{2} \tilde{\gamma}^{s}$ because of the non-unicity of the representation $\gamma=\tilde{\gamma} \cdot x$.

Also, these invariant manifolds are unique in the sense of Remark 2.4.

The main result of this section is

Theorem 7.1 (Lambda Lemma) Let $F_{\nu}$ satisfy HC1 to HC3 and HC4' with $k \geq 2$. Let $\omega_{0}$ be non-resonant. Let $\Gamma$ be an $(p+q)$-dimensional $C^{1}$ manifold intersecting transversally $W^{s}$ at $q_{0}$. Then $W^{u} \subset \overline{\cup_{n \geq 0} F^{n}(\Gamma)}$. Moreover there exist $p$-dimensional submanifolds $D$ of $\Gamma$ such that if $D_{n}$ is the connex component of $F^{n}(D) \cap B(0, \delta)$ which contains $F^{n}\left(q_{0}\right)$, for any $\varepsilon>0$ there exists $n_{0}$ such that $T D_{n}$ is $\varepsilon$-close to (a subset of) $T W^{u}$ if $n>n_{0}$.

Next subsections are devoted to prove this Theorem.

\subsection{A normal form around the invariant torus}

Although many quantities actually depend on $\nu$, in order to simplify the notation, we shall not write this dependence explicitly, unless necessary. We assume that $\mu$ is such that the conditions for the existence of the invariant manifolds hold for the $C^{2}$ case. In what follows it is not necessary to restrict $\mu$ any more.

Next lemma provides good coordinates for the local study of $F$ near $\mathbb{T}_{0}$.

Lemma 7.2 Let $F: \mathcal{M}_{\delta} \rightarrow \mathcal{M}$ be a map of the form (2.1) satisfying hypotheses HC1 to HC3 and $\mathrm{HC}_{4}$ ' for $k \geq 2$. If $\delta$ is small enough, there exists a close to the identity $C^{2}$ change of variables $C$ defined in a neighborhood of $\mathbb{T}_{0}$ such that $\tilde{F}=C^{-1} \circ F \circ C$ takes the same form (2.1)

$$
\tilde{F}(w)=\left(\tilde{A}_{-}(\theta) x, \tilde{A}_{+}(\theta) y, \theta+\tilde{\omega}(x, y, r), \tilde{B}(\theta) r\right)+\tilde{f}(w)
$$

and 
1. C restricted to $\mathbb{T}_{0}$ is the identity.

2. The stable and unstable invariant manifolds of $\mathbb{T}_{0}, W^{s}$ and $W^{u}$, are locally expressed by $\{(x, 0, \theta, 0)\}$ and $\{(0, y, \theta, 0)\}$ respectively.

3. $\tilde{f}_{3 \mid W^{u} \cup W^{s}}=0$.

4. The derivative of $\tilde{F}$ on $\mathbb{T}_{0}$ has the form

$$
D \tilde{F}(0,0, \theta, 0)=\left(\begin{array}{cccc}
\tilde{A}_{-}(\theta) & 0 & 0 & 0 \\
0 & \tilde{A}_{+}(\theta) & 0 & 0 \\
0 & 0 & I & h(\theta) \\
0 & 0 & 0 & \tilde{B}(\theta)
\end{array}\right)
$$

with $\tilde{A}_{ \pm}(\theta)=A_{ \pm}(\theta)+\mathcal{O}(\delta+L), \tilde{B}(\theta)=B(\theta)+\mathcal{O}(\delta+L)$

Proof. As we have already remarked, by Theorem 3.1 and the fact that $F$ is invertible with a suitable inverse, the local stable and unstable invariant manifolds of $\mathbb{T}_{0}, W^{s, u}$, can be represented locally in the form

$$
\begin{array}{ll}
\left(x, \gamma_{1}^{s}(x, \theta), \theta, \gamma_{2}^{s}(x, \theta)\right), & (x, \theta) \in B_{\delta}^{m} \times \mathbb{T}^{n} \\
\left(\gamma_{1}^{u}(y, \theta), y, \theta, \gamma_{2}^{u}(y, \theta)\right), & (y, \theta) \in B_{\delta}^{p} \times \mathbb{T}^{n}
\end{array}
$$

respectively. Also, the functions $\gamma_{j}^{s, u}$ verify (7.2) and

$$
\left\|D \gamma^{s, u}\right\| \leq c=\mathcal{O}(\delta+L)
$$

The first step will be to straighten the invariant manifolds, which will be achieved by means of two consecutives changes of variables. For that we first take

$$
C_{1}(x, y, \theta, r)=\left(x+\gamma_{1}^{u}(y, \theta), y, \theta, r+\gamma_{2}^{u}(y, \theta)\right) .
$$

By (7.6) we have that it is well defined in some neighborhood of $\mathbb{T}_{0}$. After the change, $W^{u}$ is given by $x=0, r=0$. Indeed, this readily follows from $C_{1}(0, y, \theta, 0)=\left(\gamma_{1}^{u}(y, \theta), y, \theta, \gamma_{2}^{u}(y, \theta)\right)$.

Since $C_{1}$ is the identity on $\mathbb{T}_{0}$ and $D C_{1}=I+\mathcal{O}(\delta+L(\delta, \mu)), C_{1}^{-1} F C_{1}$ can be written again in the form (2.1) with $\tilde{f}$ instead of $f$. Indeed from the explicit expression of $C_{1}^{-1} F C_{1}$ and the fact that $\gamma^{u}$ verifies (7.2), we have that $\tilde{f}$ verifies $\mathrm{HC} 1$ to $\mathrm{HC} 3$ and $\mathrm{HC} 4$ '.

Now let $\gamma^{s}(x, \theta)$ be the parametrization of the stable manifold in these new variables. We proceed in an analogous way to straighten the stable manifold, with the change $C_{2}(x, y, \theta, r)=$ $\left(x, y+\gamma_{1}^{s}(x, \theta), \theta, r+\gamma_{2}^{s}(x, \theta)\right)$. Notice that $C_{2}$ is the identity on the unstable manifold.

We denote again $C_{2}^{-1} \circ C_{1}^{-1} \circ F \circ C_{1} \circ C_{2}$ by $F$. We remark that it can be written in the form (2.1) verifying $\mathrm{HC} 1$ to $\mathrm{HC} 3$ and $\mathrm{HC} 4$ '.

The second step is to find a change of variables such that, in the new variables, $f_{3} \equiv 0$ on $W^{s}$. For that we look for a change $C_{3}$ having the form

$$
C_{3}(x, y, \theta, r)=(x, y, \theta+c(x, \theta), r)
$$


which conjugates $F$ restricted to $W^{s}$ to a map $\tilde{F}$ such that restricted to $W^{s}$ has the form $\tilde{F}_{\mid W^{s}}(x, \theta)=\left(\tilde{F}_{1}(x, \theta), \theta+\omega_{0}\right)$. Precisely, we ask that $\tilde{F} \circ C_{3 \mid W^{s}}=C_{3} \circ F_{\mid W^{s}}$ which gives the following condition for $c$

$$
\begin{aligned}
c(x, \theta)= & \omega(x, 0,0)-\omega_{0}+f_{3}(x, 0, \theta, 0) \\
& +c\left(A_{-}(\theta) x+f_{1}(x, 0, \theta, 0), \theta+\omega(x, 0,0)+f_{3}(x, 0, \theta, 0)\right) .
\end{aligned}
$$

We denote $\psi=\left(\psi_{1}, \psi_{2}\right)=\pi_{(x, \theta)} \circ F_{\mid W}$. We want a $C^{2}$ solution of (7.8) such that $C_{3}$ is invertible. We shall first prove that (7.8) has a $C^{1}$ solution, and then we shall prove it is $C^{2}$. We introduce the space

$$
\Sigma=\left\{c: \mathcal{B}_{\delta}^{m} \times \mathbb{T}^{n} \rightarrow \mathbb{R}^{n} ; \text { of class } C^{1}, c(0, \theta)=0,\left\|\partial_{x} c\right\|_{0},\left\|\partial_{\theta} c\right\|_{1} \text { bounded }\right\}
$$

(we recall that $\left.\left\|\partial_{\theta} c\right\|_{1}=\sup \left\|\partial_{\theta} c(x, \theta)\right\| /\|x\|\right)$ with the norm

$$
\|c\|_{\Sigma}=\alpha_{0}\left\|\partial_{x} c\right\|_{0}+\alpha_{1}\left\|\partial_{\theta} c\right\|_{1} .
$$

Let $\kappa, 0<\kappa<1$, such that $\kappa-\left(\lambda^{-1}+L\right)\left(1+K_{\theta}\right)^{2}>0$. We choose $\alpha_{0}, \alpha_{1}$ satisfying the condition

$$
\frac{K_{l}+K_{m}}{\kappa-\left(\lambda^{-1}+L\right)}<\frac{\alpha_{0}}{\alpha_{1}}<\frac{1}{\delta} \frac{\kappa-\left(\lambda^{-1}+L\right)\left(1+K_{\theta}\right)}{\left(\lambda^{-1}+L\right)\left(K_{\omega}+K_{f}\right)} .
$$

Notice that if $\delta$ is small, (7.9) can be satisfied. We define $G$ to be the right hand side of (7.8).

Clearly $G$ maps $\Sigma$ to $\Sigma$ : the condition $\partial_{\theta}[G(c)] /\|x\|$ bounded follows from HC3 and HC4' which implies that $\partial_{\theta} f_{j}(x, 0, \theta, 0) /\|x\|, j=1,3$, are bounded.

Now we see that $G$ is a contraction:

$$
\begin{aligned}
& \left\|G\left(c_{2}\right)-G\left(c_{1}\right)\right\|_{\Sigma} \leq \alpha_{0}\left\|\partial_{x}\left[G\left(c_{2}\right)\right]-\partial_{x}\left[G\left(c_{1}\right)\right]\right\|_{0}+\alpha_{1}\left\|\partial_{\theta}\left[G\left(c_{2}\right)\right]-\partial_{\theta}\left[G\left(c_{1}\right)\right]\right\|_{1} \\
& \leq \quad \alpha_{0}\left(\left(\lambda^{-1}+L\right)+\alpha_{0}^{-1} \alpha_{1}\left(K_{l}+K_{m}\right)\right)\left\|\partial_{x} c_{1}-\partial_{x} c_{2}\right\|_{0} \\
& \quad+\alpha_{1}\left(\left(1+K_{\theta}\right)\left(\lambda^{-1}+L\right)+\alpha_{0} \alpha_{1}^{-1}\left(K_{\omega}+K_{f}\right)\left(\lambda^{-1}+L\right) \delta\right)\left\|\partial_{\theta} c_{1}-\partial_{\theta} c_{2}\right\|_{1} \\
& \quad \leq \quad \kappa\left\|c_{2}-c_{1}\right\|_{\Sigma} .
\end{aligned}
$$

Next we see that the fixed point of $G$ is in fact $C^{2}$. We consider the space

$D \Sigma=\left\{\varphi=\left(\varphi_{0}, \varphi_{1}, \varphi_{2}\right) ; \varphi_{j} \in C^{0}\left(\mathcal{B}_{\delta}^{m} \times \mathbb{T}^{n}, L^{2-j}\left(\mathbb{R}^{m}, L^{j}\left(\mathbb{R}^{n}, \mathbb{R}^{m}\right)\right)\right),\left\|\varphi_{0}\right\|_{0},\left\|\varphi_{1}\right\|_{0},\left\|\varphi_{2}\right\|_{1}<\infty\right\}$

with the norm $\|\varphi\|_{D \Sigma}=a_{0}\left\|\varphi_{0}\right\|_{0}+a_{1}\left\|\varphi_{1}\right\|_{0}+a_{2}\left\|\varphi_{2}\right\|_{1}$. Let $\kappa$ be as before and $\delta$ such that $\left(1+K_{\theta}\right)\left(\lambda^{-1}+L\right)+\left(K_{\omega}+K_{f}\right)\left(K_{l}+K_{m}\right) \delta<\kappa$. We choose $a_{0}, a_{1}, a_{2}$ positive numbers satisfying

$$
\begin{aligned}
& a_{0}>\frac{a_{1}\left(K_{l}+K_{m}\right)\left(\lambda^{-1}+L\right) \delta+a_{2}\left(K_{l}+K_{m}\right)^{2} \delta}{\kappa-\left(\lambda^{-1}+L\right)^{2}} \\
& a_{1}>2 \frac{a_{0}\left(K_{\omega}+K_{f}\right)\left(\lambda^{-1}+L\right)+a_{2}\left(1+K_{\theta}\right)\left(K_{l}+K_{m}\right)}{\kappa-\left(1+K_{\theta}\right)\left(\lambda^{-1}+L\right)+\left(K_{\omega}+K_{f}\right)\left(K_{l}+K_{m}\right) \delta} \\
& a_{2}>\frac{\left(a_{0}\left(K_{\omega}+K_{f}\right)^{2}+a_{1}\left(1+K_{\theta}\right)\left(K_{\omega}+K_{f}\right)\right)\left(\lambda^{-1}+L\right) \delta}{\kappa-\left(1+K_{\theta}\right)^{2}\left(\lambda^{-1}+L\right)}
\end{aligned}
$$


( $a_{0}, a_{1}, a_{2}$ may be chosen verifying the second condition. Making $\delta$ smaller if necessary, the other two conditions are fulfilled.) We look for $H$ such that

$$
H\left(c, \partial_{x}^{2} c, \partial_{x \theta} c, \partial_{\theta}^{2} c\right)=\left(\partial_{x}^{2}[G(c)], \partial_{x \theta}[G(c)], \partial_{\theta}[G(c)]\right) .
$$

We take $H=\left(H_{0}, H_{1}, H_{2}\right)$ with

$$
\begin{aligned}
H_{0}= & \partial_{x}^{2} \omega+\partial_{x}^{2} f_{3}+\partial_{x} c \circ \psi \cdot \partial_{x}^{2} f_{1}+\partial_{\theta} c \circ \psi \cdot\left(\partial_{x}^{2} \omega+\partial_{x}^{2} f_{3}\right)+\varphi_{0} \circ \psi \cdot\left(A_{-}+\partial_{x} f_{1}\right)^{2} \\
& +2 \varphi_{1} \circ \psi \cdot\left(\partial_{x} \omega+\partial_{x} f_{3}\right)\left(A_{-}+\partial_{x} f_{1}\right)+\varphi_{2} \circ \psi \cdot\left(\partial_{x} \omega+\partial_{x} f_{3}\right)^{2}, \\
H_{1}= & \partial_{x \theta} f_{3}+\partial_{x} c \circ \psi \cdot\left(\partial_{\theta} A_{-}+\partial_{\theta x} f_{1}\right)+\partial_{\theta} c \circ \psi \cdot \partial_{\theta x} f_{3}+\varphi_{0} \circ \psi \cdot\left(\partial_{\theta} A_{-} x+\partial_{\theta} f_{1}\right)\left(A_{-}+\partial_{x} f_{1}\right) \\
& +\varphi_{1} \circ \psi \cdot\left(\left(I+\partial_{\theta} f_{3}\right)\left(A_{-}+\partial_{x} f_{1}\right)+\left(\partial_{\theta} A_{-} x+\partial_{\theta} f_{1}\right)\left(\partial_{x} \omega+\partial_{x} f_{3}\right)\right) \\
& +\varphi_{2} \circ \psi \cdot\left(\partial_{x} \omega+\partial_{x} f_{3}\right)\left(I+\partial_{\theta} f_{3}\right), \\
H_{2}= & \partial_{\theta}^{2} f_{3}+\partial_{x} c \circ \psi \cdot\left(\partial_{\theta}^{2} A_{-} x+\partial_{\theta}^{2} f_{1}\right)+\partial_{\theta} c \circ \psi \cdot \partial_{\theta}^{2} f_{3}+\varphi_{0} \circ \psi \cdot\left(\partial_{\theta} A_{-} x+\partial_{\theta} f_{1}\right)^{2} \\
& +2 \varphi_{1} \circ \psi \cdot\left(I+\partial_{\theta} f_{3}\right)\left(\partial_{\theta} A_{-} x+\partial_{\theta} f_{1}\right)+\varphi_{2} \circ \psi \cdot\left(I+\partial_{\theta} f_{3}\right)^{2} .
\end{aligned}
$$

We have that $H$ is a well defined map from $\Sigma \times D \Sigma$ to itself. Indeed, since $\partial_{\theta} f_{j}(x, 0, \theta, 0) /\|x\|$ and $\partial_{\theta}^{2} f_{j}(x, 0, \theta, 0) /\|x\|$ are bounded for $j=1,3$, if $(c, \varphi) \in \Sigma \times D \Sigma$, then $H_{2}(c(x, \theta), \varphi(x, \theta)) /\|x\|$ is bounded. Also for each $\varphi \in D \Sigma, H(\cdot, \varphi)$ is continuous. Indeed, the continuity of $H_{0}$ and $H_{1}$ is obvious. For $H_{2}$ we have

$$
\left\|H_{2}\left(c^{\prime}, \varphi\right)-H_{2}(c, \varphi)\right\|_{1} \leq\left\|\partial_{x} c^{\prime}-\partial_{x} c\right\|_{0} \sup \left(\left\|\partial_{\theta}^{2} A_{-} x+\partial_{\theta}^{2} f_{1}\right\|\|x\|^{-1}\right)+\left\|\partial_{\theta} c^{\prime}-\partial_{\theta} c\right\|_{1}\left\|\partial_{\theta}^{2} f_{3}\right\|,
$$

which is small if $\left\|c^{\prime}-c\right\|_{\Sigma}$ is small. Finally, we check that $H(c, \cdot)$ is a uniform contraction in $D \Sigma$ :

$$
\begin{aligned}
& \left\|H\left(c, \varphi^{\prime}\right)-H(c, \varphi)\right\|_{D \Sigma} \\
& \leq a_{0}\left(\left(\lambda^{-1}+L\right)^{2}+a_{0}^{-1} a_{1}\left(K_{l}+K_{m}\right)\left(\lambda^{-1}+L\right) \delta+a_{0}^{-1} a_{2}\left(K_{l}+K_{m}\right)^{2} \delta\right)\left\|\varphi_{0}^{\prime}-\varphi_{0}\right\|_{0} \\
& +a_{1}\left(\left(1+K_{\theta}\right)\left(\lambda^{-1}+L\right)+\left(K_{\omega}+K_{f}\right)\left(K_{l}+K_{m}\right) \delta\right. \\
& \left.+a_{1}^{-1} a_{0} 2\left(K_{\omega}+K_{f}\right)\left(\lambda^{-1}+L\right)+a_{1}^{-1} a_{2} 2\left(1+K_{\theta}\right)\left(K_{l}+K_{m}\right)\right)\left\|\varphi_{1}^{\prime}-\varphi_{1}\right\|_{0} \\
& +a_{2}\left(\left(1+K_{\theta}\right)^{2}\left(\lambda^{-1}+L\right)+a_{2}^{-1} a_{0}\left(K_{\omega}+K_{f}\right)^{2}\left(\lambda^{-1}+L\right) \delta\right. \\
& \left.+a_{2}^{-1} a_{1}\left(1+K_{\theta}\right)\left(K_{\omega}+K_{f}\right)\left(\lambda^{-1}+L\right) \delta\right)\left\|\varphi_{2}^{\prime}-\varphi_{2}\right\|_{1} \\
& \leq \kappa\left\|\varphi^{\prime}-\varphi\right\|_{D \Sigma} \text {. }
\end{aligned}
$$

Then we define the map $\Gamma=(G, H): \Sigma \times D \Sigma \rightarrow \Sigma \times D \Sigma$. Applying the Fiber Contraction Theorem to $\Gamma$ as indicated in Section 4, we have that $c$, the fixed point of the map $G$, is of class $C^{2}$ and their second derivatives belong to $D \Sigma$.

To get $\tilde{f}_{3} \equiv 0$ on $W^{u} \mathbb{T}_{0}$ we consider a new change $C_{4}$ of the form $C_{4}(x, y, \theta, r)=(x, y, \theta+$ $c(y, \theta), r)$, with $c(0, \theta)=0$. We impose that $\tilde{F}^{-1} \circ C_{4 \mid W^{u}}=C_{4} \circ F^{-1} \mid W^{u}$, where $\tilde{F}_{\mid W^{u}}^{-1}(x, \theta)=$ $\left(\tilde{F}_{1}^{-1}(x, \theta), \theta-\omega\right)$.

The new $\tilde{f}_{3}$ also verifies $\tilde{f}_{3} \equiv 0$ on $W^{s}$ because $C_{4}$ is the identity on $W^{s}$.

Up to now, we have that

$$
D \tilde{F}(0,0, \theta, 0, \nu)=\left(\begin{array}{cccc}
\tilde{A}_{-}(\theta) & 0 & 0 & h_{1}(\theta) \\
0 & \tilde{A}_{+}(\theta) & 0 & h_{2}(\theta) \\
0 & 0 & I & h_{3}(\theta) \\
0 & 0 & 0 & \tilde{B}(\theta)
\end{array}\right) .
$$


Following Lemma 6.4, which is proved by induction on the degree of differentiability, there exists a $C^{2}$ change change of the form $C_{5}(x, y, \theta, r)=\left(x+a_{1}(\theta) r, y+a_{2}(\theta) r, \theta, r\right)$ such that $D \tilde{F}(0,0, \theta, 0)$ has the desired form. In this case the situation is simpler because the equations for $a_{1}$ and $a_{2}$ are

$$
\tilde{A}_{-} a_{1}+h_{1}=a_{1}^{*} \tilde{B}, \quad \tilde{A}_{+} a_{2}+h_{2}=a_{2}^{*} \tilde{B},
$$

which are linear and uncoupled.

\subsection{Lambda Lemma}

This section is devoted to prove Theorem 7.1. We shall call p-dimensional disk to the image of a p-dimensional ball into $\mathcal{M}$ by a $C^{1}$ map. The theorem readily follows from

Proposition 7.3 Let $F$ be as in Lemma (7.2). Assume that $\omega_{0}=\omega(0,0,0)$ is non-resonant. Let $p_{0} \in W^{u}$ and $\Gamma$ a $C^{1}(p+q)$-dimensional manifold transversal to $W^{s}$ on $q_{0}$. Then there exists a p-dimensional disk contained in $\Gamma$, given by $q(z)=(x(z), z, \theta(z), r(z)), z \in B_{\zeta_{0}}=$ $\left\{z ;\|z\|<\zeta_{0}\right\} \subset \mathbb{R}^{p}$, with $q(0)=q_{0}$, such that

$$
\forall \varepsilon>0 \quad \exists j \in \mathbb{N} \quad \text { such that } \quad\left\{F^{j}(q(z)) ; z \in B_{\zeta_{0}}\right\} \cap B\left(p_{0}, \varepsilon\right) \neq \emptyset .
$$

Furthermore there exists $j_{0}$ such that $D\left[F^{j}(q(z))\right]\left(\mathbb{R}^{p}\right)$ is $\varepsilon$-close to a subspace of $T W^{u}$ for $j>j_{0}$.

The proof is very similar to the analogous version in [10] where the case of $x, y$ onedimensional and $F$ analytic is considered. We follow more or less this proof.

Proof. Let $U_{\rho}=\{(x, y, \theta, r) ;\|x\|,\|y\|,\|r\|<\rho\}$ be a neighborhood of $\mathbb{T}_{0}$ where the coordinates given by Lemma 7.2 are defined, $\rho>0$ to be fixed later, and $F$ be expressed in these coordinates. Let $\varepsilon>0$. It is not restrictive to suppose that $\varepsilon<1 / 2$.

We can suppose that $p_{0}$ and $q_{0}$ are in $U_{\rho}$, iterating by $F^{-1}$ and $F$ respectively, if necessary. Therefore we can write $p_{0}=\left(0, y_{0}, \varphi_{0}, 0\right)$ and $q_{0}=\left(x_{0}, 0, \theta_{0}, 0\right)$, with $\left\|x_{0}\right\|$ and $\left\|y_{0}\right\|$ as small as necessary, but otherwise fixed. On $U_{\rho}, D F$ takes the form

$$
\left(\begin{array}{cccc}
A_{-}+a_{11} & a_{12} & a_{13} & a_{14} \\
a_{21} & A_{+}+a_{22} & a_{23} & a_{24} \\
a_{31} & a_{32} & I+a_{33} & a_{34} \\
a_{41} & a_{42} & a_{43} & B+a_{44}
\end{array}\right)
$$

with $\left\|A_{-}(\theta)\right\|<\lambda^{-1},\left\|A_{+}^{-1}(\theta)\right\|<\lambda^{-1},\|B(\theta)\|<\beta$ and $a_{i j}$ are $C^{1}$ with $a_{i j}(0,0, \theta, 0)=0$, $(i, j) \neq(3,4)$.

Let $\lambda_{0}$ be such that

$$
\beta<\lambda_{0}<\lambda \text {. }
$$

Given $\eta_{0}>0$, let $\kappa>0$ be such that

$$
\begin{aligned}
& \beta+3 \kappa<\lambda_{0}<\lambda-\kappa\left(1+\eta_{0}\right), \\
& \lambda_{0}<\lambda-7 \kappa / 4, \\
& 0<\frac{\kappa+(\beta+\kappa) \eta_{0}}{\lambda_{0}}<\eta_{0}, \\
& \lambda^{-1}+2 \kappa<1 .
\end{aligned}
$$


Now we can arrange things so that

$$
\begin{aligned}
\left\|a_{i j}\right\| & \leq \kappa, \quad|i-3|+|j-4| \neq 0 \\
\left\|a_{34}\right\| & \leq 1 \\
\left\|a_{i 2}\right\| & \leq\|(x, r)\|
\end{aligned}
$$

Indeed, since the local unstable manifold is represented by $\{x=0, r=0\}$ and $\omega+f_{3} \equiv \omega_{0}$ over it, we have

$$
f_{1}(0, y, \theta, 0)=0, \quad f_{4}(0, y, \theta, 0)=0, \quad \omega(0, y, 0)+f_{3}(0, y, \theta, 0)=\omega_{0}
$$

Hence the derivatives of $f_{1}, f_{4}$ and $\omega+f_{3}$ with respect to $y$ and $\theta$ are zero on $\{x=0, r=0\}$. Then, there exists $K_{a}>0$ such that $\left\|a_{12}\right\|,\left\|a_{32}\right\|,\left\|a_{42}\right\|<K_{a}\|(x, r)\|$. On the other hand, by hypothesis we have that $\left\|a_{34}\right\|<K_{\omega}+K_{f}$.

Next, given $\alpha>0$, we consider the directional scaling $g(x, y, \theta, r)=(\alpha x, \alpha y, \theta, \alpha r)$. After the scaling, $F$ is defined on $U_{\rho / \alpha},\left\|a_{34}\right\| \leq \alpha\left(K_{\omega}+K_{3}\right)$ and $\left\|a_{i 2}\right\| \leq \alpha K_{a}\|(x, r)\|, i=1,3,4$. We choose $\alpha$ such that $\alpha\left(K_{\omega}+K_{f}\right) \leq 1$ and $\alpha K_{a} \leq 1$.

Finally, taking $\rho$ smaller, if necessary, we can assume, by 4) of Lemma 7.2 that, except for $\left\|a_{34}\right\|$, we have $\left\|a_{i j}\right\|<\kappa$ on $U$.

We further introduce $\tau$ such that

$$
1<\tau, \quad 0<\frac{2}{\lambda_{0}-(\beta+3 \kappa)}<\tau, \quad \frac{4 K_{l}}{\lambda-\lambda_{0}-7 \kappa / 4}<\tau, \quad \frac{1}{\tau}<\rho .
$$

Since, as we have said, we can take $p_{0}=\left(0, y_{0}, \varphi_{0}, 0\right)$ as near to the torus as we want, we take $p_{0}$ with $\left\|y_{0}\right\|<1 / \tau$. Notice that $\tau$ is independent of $\varepsilon$.

Moreover, using the invariance conditions $f_{2}(x, 0, \theta, 0)=0, f_{4}(x, 0, \theta, 0)=0$, and the condition $\omega(x, 0,0)+f_{3}(x, 0, \theta, 0)=\omega_{0}$, we have that

$$
D F_{\mid W s \cap U}=\left(\begin{array}{cccc}
A_{-}+a_{11} & a_{12} & a_{13} & a_{14} \\
0 & A_{+}+a_{22} & 0 & a_{24} \\
0 & a_{32} & I & a_{34} \\
0 & a_{42} & 0 & B+a_{44}
\end{array}\right) .
$$

Since in the local variables

$$
T_{q_{0}} W^{s}=\left\{v=\left(v_{x}, v_{y}, v_{\theta}, v_{r}\right) \in \mathbb{R}^{m} \times \mathbb{R}^{p} \times \mathbb{R}^{n} \times \mathbb{R}^{q} ; v_{y}=0, v_{r}=0\right\}
$$

and $\Gamma$ intersects transversally $W^{s}$ there exists a $p$-disk in $\Gamma, q_{0}(z)=\left(x_{0}(z), z, \theta_{0}(z), r_{0}(z)\right)$, $z \in B_{\zeta_{0}}$, with $q_{0}(0)=q_{0}, q_{0}^{\prime}(0)=\left(x_{0}^{\prime}, I, \theta_{0}^{\prime}, r_{0}^{\prime}\right)^{t}$ and $\left\|r_{0}^{\prime}\right\| \leq \eta_{0}$. We are going to iterate the disk $q_{0}(z)$ by $F$ in two steps: first we iterate until its tangent space at $z=0$ is close enough to the space $\left\{v_{x}=0, v_{\theta}=0, v_{r}=0\right\}$. Then we continue iterating until the disk is close enough to $W^{u}$.

We consider

$$
\bar{q}_{k+1}=F \circ q_{k}, \quad q_{k+1}=\bar{q}_{k+1} \circ \mathcal{R}_{k+1}, \quad \mathcal{R}_{k+1}(z)=\left(\bar{y}_{k+1}^{\prime}(0)\right)^{-1} z
$$

(in words, $q_{k+1}$ is a reparametrization of the image of $q_{k}$ by $F$ so that $y_{k+1}^{\prime}(0)=I$.) 
Since $q_{k}(0)=\left(x_{k}(0), 0, \theta_{k}(0), 0\right)=F^{k}\left(q_{0}\right)$ tend to the torus, the disks are defined for $z$ small. Let $A_{ \pm}^{k}=A_{ \pm}\left(\theta_{k}\right), B^{k}=B\left(\theta_{k}\right)$ and $a_{i j}^{k}=a_{i j}\left(q_{k}\right)$. We define $\kappa_{k}=\max \left\{\left\|a_{i j}^{k}\right\|, j \neq 4\right\}$. We have that $\kappa_{k} \leq \kappa$ and $\kappa_{k} \rightarrow 0$. By (7.16) we can write

$$
\bar{q}_{k+1}^{\prime}(0)=D F\left(q_{k}(0)\right) q_{k}^{\prime}(0)=\left(\begin{array}{l}
\left(A_{-}^{k}+a_{11}^{k}\right) x_{k}^{\prime}+a_{12}^{k}+a_{13}^{k} \theta_{k}^{\prime}+a_{14}^{k} r_{k}^{\prime} \\
A_{+}^{k}+a_{22}^{k}+a_{24}^{k} r_{k}^{\prime} \\
a_{32}^{k}+\theta_{k}^{\prime}+a_{34}^{k} r_{k}^{\prime} \\
a_{42}^{k}+\left(B^{k}+a_{44}^{k}\right) r_{k}^{\prime}
\end{array}\right)
$$

and therefore we have the recurrences

$$
\begin{aligned}
x_{k+1}^{\prime} & =\left(\left(A_{-}+a_{11}\right) x_{k}^{\prime}+a_{12}+a_{13} \theta_{k}^{\prime}+a_{14} r_{k}^{\prime}\right)\left(A_{+}+a_{22}+a_{24} r_{k}^{\prime}\right)^{-1} \\
y_{k+1}^{\prime} & =I \\
\theta_{k+1}^{\prime} & =\left(a_{32}+\theta_{k}^{\prime}+a_{34} r_{k}^{\prime}\right)\left(A_{+}+a_{22}+a_{24} r_{k}^{\prime}\right)^{-1} \\
r_{k+1}^{\prime} & =\left(a_{42}+\left(B+a_{44}\right) r_{k}^{\prime}\right)\left(A_{+}+a_{22}+a_{24} r_{k}^{\prime}\right)^{-1}
\end{aligned}
$$

We introduce $m_{k}=\max \left\{\left\|x_{k}^{\prime}\right\|,\left\|\theta_{k}^{\prime}\right\|\right\}$ and $\eta_{k}=\left\|r_{k}^{\prime}\right\|$. We claim that the following inequalities hold:

$$
\begin{aligned}
m_{k+1} & \leq \frac{m_{k}}{\lambda_{0}}+\frac{\kappa_{k}}{\lambda_{0}}+\frac{\kappa}{\lambda_{0}} \eta_{k} \\
\eta_{k+1} & \leq \frac{\kappa_{k}}{\lambda_{0}}+\frac{\beta+\kappa}{\lambda_{0}} \eta_{k}, \\
\eta_{k+1} & \leq \eta_{0}
\end{aligned}
$$

We prove $(7.17),(7.18)$ and $(7.19)$ by induction. First we have to see that

$$
\bar{y}_{k+1}^{\prime}(0)=A_{+}^{k}+a_{22}^{k}+a_{24}^{k} r_{k}^{\prime}
$$

is invertible. It follows from $\left\|a_{22}+a_{24} r_{k}^{\prime}\right\|<\left\|A_{+}^{-1}\right\|^{-1}$. We also have that $\|\left(A_{+}+a_{22}+\right.$ $\left.a_{24} r_{k}^{\prime}\right)^{-1}\|\leq\| A_{+}^{-1} \| /\left(1-\left\|A_{+}^{-1}\right\|\left\|a_{22}+a_{24} r_{k}^{\prime}\right\|\right) \leq 1 /\left(\lambda-\kappa_{k}-\kappa \eta_{k}\right) \leq \lambda_{0}^{-1}$.

Then (7.17) and (7.18) follows at once from the recurrences and (7.19) follows from (7.13).

By the conditions on $\kappa, \eta_{0}$ and $\lambda$ we can apply the following property of sequences of real numbers, first to $\eta_{k}$ and then to $m_{k}$, to conclude that both sequences go to zero:

Lemma 7.4 Let $\left(a_{k}\right),\left(b_{k}\right),\left(\xi_{k}\right)$ be sequences of positive real numbers such that $a_{k} \rightarrow 0$, $b_{k} \leq b<1$ and $\xi_{k+1} \leq a_{k}+b_{k} \xi_{k}, k \geq 1$. Then $\xi_{k} \rightarrow 0$.

Therefore, there exists $k_{0}$ such that if $k \geq k_{0}$, then $m_{k}, \eta_{k}<\varepsilon / 4$ and $\left\|x_{k}(0)\right\|<\varepsilon /(2 \tau)$. We consider the disk $q_{k_{0}}(z)$ and we reparametrize it in such a way that $y_{k_{0}}(z)=I$. This is possible, locally at $z=0$, because $y_{k_{0}}^{\prime}(0)=I$. This does not change the bounds $m_{k_{0}}$ and $\eta_{k_{0}}$.

We denote again by $q_{0}(z)$ the resulting disk $q_{k_{0}}(z)$.

Now we perform the second step where we iterate this new $q_{0}(z), z \in B_{\zeta_{0}}$, with $\zeta_{0}$ chosed below.

We consider again the sequence

$$
\bar{q}_{k+1}=F \circ q_{k}, \quad q_{k+1}=\bar{q}_{k+1} \circ \mathcal{R}_{k+1}, \quad \mathcal{R}_{k+1}=\left(\bar{y}_{k+1}\right)^{-1}
$$


with $q_{k+1}$ defined for $\|z\|<\zeta_{k+1}=\min \left(\lambda_{0} \zeta_{k}, 1 / \tau\right)$, but note that now $\mathcal{R}_{k+1}$ is not linear and we have $y_{k+1}^{\prime}(z)=I, \forall z \in B_{\zeta_{k+1}}$. Let

$$
m_{k}=\sup _{z \in B_{\zeta_{k}}}\left\{\left\|x_{k}^{\prime}(z)\right\|,\left\|\theta_{k}^{\prime}(z)\right\|,\left\|r_{k}^{\prime}(z)\right\|\right\} .
$$

We consider the neighborhood of $W^{u}$ defined by $V_{\varepsilon / \tau}=\left\{(x, y, \theta, r) \in U_{\rho / \alpha} ;\|(x, r)\|<\varepsilon / \tau\right\}$.

$\zeta_{0}$ is selected so that $m_{0}<\frac{\varepsilon}{2}$, which is possible by the continuity of $q_{0}(z)$ and its derivatives, and that $q_{0}(z) \in V_{\varepsilon / \tau}$, for $z \in B_{\zeta_{0}}$.

We check inductively that $q_{k+1}$ is well defined, it is contained in $V_{\varepsilon / \tau}$ and that $m_{k}<\varepsilon / 2$. First we prove that $\bar{y}_{k+1}(z)$ is invertible, $\bar{y}_{k+1}\left(B_{\zeta_{k}}\right) \supset B_{\lambda_{0} \zeta_{k}}$ and

$$
\left\|\left(\bar{y}_{k+1}^{-1}\right)^{\prime}(z)\right\|<\lambda_{0}^{-1}, \quad z \in B_{\lambda_{0} \zeta_{k}} .
$$

We write $\bar{y}_{k+1}=h_{1}+h_{2}$ with

$$
h_{1}(z)=A_{+}\left(\theta_{k}(z)\right) z
$$

and

$$
h_{2}(z)=f_{2}\left(q_{k}(z)\right) .
$$

It is easy to see that $h_{1}$ is invertible, $h_{1}\left(B_{\zeta_{k}}\right) \supset B_{\lambda \zeta_{k}}$ and

$$
\operatorname{Lip}\left(h_{1}^{-1}\right)<\frac{1}{\lambda-\zeta_{k} K_{l} \varepsilon / 2} \text {. }
$$

On the other hand Lip $h_{2} \leq \sup \| \frac{d}{d z}\left[f_{2}\left(q_{k}(z)\right] \| \leq \kappa(1+3 \varepsilon / 2)\right.$. Therefore, combining Theorem 1.5 and Proposition 1.7 of [12] we get that $h_{1}+h_{2}$ is invertible, $\left(h_{1}+h_{2}\right)^{-1}$ is defined on $B_{\zeta_{k+1}^{*}}$ with $\zeta_{k+1}^{*}=\zeta_{k}\left(\lambda-\varepsilon K_{l} /(2 \tau)\right)\left(1-\frac{1}{\lambda-\varepsilon K_{l} /(2 \tau)} \kappa(1+3 \varepsilon / 2)\right)$. From (7.15) we have that $\zeta_{k+1}^{*} \geq \zeta_{k} \lambda_{0}$, and

$$
\operatorname{Lip}\left(h_{1}+h_{2}\right)^{-1} \leq \frac{1}{\left.\lambda-\varepsilon K_{l} /(2 \tau)-\kappa(1+3 \varepsilon / 2)\right)} \leq 1 / \lambda_{0} .
$$

By the definition of $m_{k}$ we have that

$$
m_{k+1} \leq \frac{(\beta+3 \kappa) m_{k}}{\lambda_{0}}+\frac{\varepsilon}{\tau} \frac{1}{\lambda_{0}}
$$

where we have used $\left\|a_{i 2}\right\| \leq\|(x, r)\|$. From (7.20) and (7.15), if $m_{k}<\varepsilon / 2$ then $m_{k+1}<\varepsilon / 2$.

Finally we must check that $q_{k+1}(z) \in V_{\varepsilon / \tau}$, for $\|z\|<\zeta_{k+1}$. For that we bound $\| x_{k+1}(z)-$ $x_{k+1}(0) \|$ and $\left\|r_{k+1}(z)-r_{k+1}(0)\right\|$ by using the Mean Value Theorem and we take into account that $\left\|x_{k+1}(0)\right\|<\varepsilon /(2 \tau)$ and $r_{k+1}(0)=0$. Since $m_{k+1}<\varepsilon / 2$ the result follows.

Since $\lambda_{0}^{k} \zeta_{0}$ increases geometrically we can suppose that there exists $k_{1}$ such that $\zeta_{k}=1 / \tau$ for $k \geq k_{1}$

We recall that $p_{0}=\left(0, y_{0}, \varphi_{0}, 0\right)$. As $q_{k}(0)$ tends to the torus and the rotation on the torus is irrational, there exists some large enough $k_{2} \geq k_{1}$ such that $\left\|\theta_{k_{1}}(0)-\varphi_{0}\right\|<\varepsilon / 2$. Then taking $z=y_{0}<1 / \tau$

$$
\begin{aligned}
\left\|q_{k_{1}}\left(y_{0}\right)-p_{0}\right\| & =\left\|\left(x_{k_{1}}\left(y_{0}\right), 0, \theta_{k_{1}}\left(y_{0}\right)-\varphi_{0}, r_{k_{1}}\left(y_{0}\right)\right)\right\| \\
& \leq \max \left\{\frac{\varepsilon}{\tau},\left\|\theta_{k_{1}}\left(y_{0}\right)-\theta_{k_{1}}(0)\right\|+\left\|\theta_{k_{1}}(0)-\varphi_{0}\right\|\right\} \\
& \leq \max \left\{\frac{\varepsilon}{\tau}, \frac{\varepsilon}{2} \frac{1}{\tau}+\frac{\varepsilon}{2}\right\}<\varepsilon .
\end{aligned}
$$

The statement about the $C^{1}$ closeness follows because $m_{k}<\varepsilon / 2$. 


\section{Aknowledgements}

The authors acknowledge the partial support of the the Spanish Grant DGICYT, PB94-0215 and the Catalan Grant CIRIT 1998 SGR-00041.

\section{References}

[1] Arnold V I 1964 Instability of dynamical systems with several degrees of freedom Soviet Math. Dokl. 5 581-585

[2] Bolotin S Treschev D 1999 Unbounded growth of energy in nonautonomous Hamiltonian systems Nonlinearity $12365-388$

[3] Chierchia L and Gallavotti G 1994 Drift and diffusion in phase space Ann. Int. H. Poincaré Phys. Th. 60 1-144

[4] Cresson J 1997 A $\lambda$-lemma for partially hyperbolic tori and the obstruction property Letters in Math. Phys. 42 363-377

[5] Delshams A, de la Llave R and Martínez-Seara T 1998 A geometric approach to the existence of orbits with unbounded energy in generic periodic perturbations by potential of generic geodesic flows on $\mathbb{T}^{2}$ to appear in Comm. Math. Phys.

[6] Douady R 1988 Stabilité ou instabilité des points fixes elliptiques Annal. Sci. de l'ENS $211-46$

[7] Douady D and Le Calvez P 1982 Exemple de point fixe elliptique non topologiquement stable en dimension 4 Problèmes Mathématiques de la Mécanique, C.R. Acad. Sc. Paris $296895-898$

[8] Eliasson L H 1994 Biasymptotic solutions of perturbed integrable Hamiltonian systems Bol. Soc. Bras. Mat. 25 57-76

[9] Fontich E and Martín P 1998 Arnold diffusion in perturbations of analytic exact symplectic maps to appear in Nonlinear Analysis, TMA

[10] Fontich E and Martín P 1999 Arnold diffusion in perturbations of analytic integrable Hamiltonian systems preprint

[11] Graff S 1974 On the conservation of hyperbolic invariant tori for hamiltonian systems $J$. Differential Equations 15 1-69

[12] Hirsch M W and Pugh C C 1970 Stable Manifolds and Hyperbolic Sets Proc. Symp. in Pure Math 14 133-164

[13] Hirsch M W Pugh C C and Shub M 1977 Invariant manifolds Lecture Notes in Mathematics (Berlin: Springer)

[14] de la Llave R and Wayne C E 1989 Whiskered and low dimensional tori in nearly integrable Hamiltonian systems manuscript 
[15] Marco J P 1996 Transition le long des chaînes de tores invariants pour les systèmes hamiltoniens analytiques Annales de l'I.H.P., Physique Théorique 64 205-252

[16] Palis N and de Melo W 1982 Geometric Theory of Dynamical Systems (New York: Springer)

[17] Treshev D V 1991 The mechanism of destruction of resonance tori of Hamiltonian systems Math USSR Sbornik 68 181-203

[18] Wiggins S 1988 Global bifurcations and chaos (New York: Springer)

[19] Zehnder E 1976 Generalized implicit function theorems with applications to some small divisor problems, II Comm. Pure Appl. Math. 29 49-111 